\title{
A Model for Minimizing Active Processor Time
}

\author{
Jessica Chang* Harold N. Gabow ${ }^{\dagger} \quad$ Samir Khuller ${ }^{\ddagger}$
}

\begin{abstract}
We introduce the following elementary scheduling problem. We are given a collection of $n$ jobs, where each job $J_{i}$ has an integer length $\ell_{i}$ as well as a set $T_{i}$ of time intervals in which it can be feasibly scheduled. Given a parameter $B$, the processor can schedule up to $B$ jobs at a timeslot $t$ so long as it is "active" at $t$. The goal is to schedule all the jobs in the fewest number of active timeslots. The machine consumes a fixed amount of energy per active timeslot, regardless of the number of jobs scheduled in that slot (as long as the number of jobs is non-zero). In other words, subject to $\ell_{i}$ units of each job $i$ being scheduled in its feasible region and at each slot at most $B$ jobs being scheduled, we are interested in minimizing the total time during which the machine is active. We present a linear time algorithm for the case where jobs are unit length and each $T_{i}$ is a single interval. For general $T_{i}$, we show that the problem is $N P$-complete even for $B=3$. However when $B=2$, we show that it can be efficiently solved. In addition, we consider a version of the problem where jobs have arbitrary lengths and can be preempted at any point in time. For general $B$, the problem can be solved by linear programming. For $B=2$, the problem amounts to finding a triangle-free 2-matching on a special graph. We extend the algorithm of Babenko et. al. 5] to handle our variant, and also to handle non-unit length jobs. This yields an $O(\sqrt{L} m)$ time algorithm to solve the preemptive scheduling problem for $B=2$, where $L=\sum_{i} \ell_{i}$. We also show that for $B=2$ and unit length jobs, the optimal non-preemptive schedule has $\leq 4 / 3$ times the active time of the optimal preemptive schedule; this bound extends to several versions of the problem when jobs have arbitrary length.
\end{abstract}

\section{Introduction}

Power management strategies have been widely studied in the scheduling literature [1, 2, 41, 59, 40, 7]. Many of the models are motivated by the energy consumption of the processor. Consider, alternatively, the energy consumed by the operation of large storage systems. Data is stored in memory which may be turned on and off [4], and each task or job needs to access a subset of data items to run. At each time step, the scheduler can work on a group of at most $B$ jobs. The only requirement is that the memory banks containing the required data from these jobs be turned on. The problem studied in this paper is the special case where all the data is in one memory bank. For even special cases involving multiple memory banks, the problem becomes NP-complete: if each job needs access to multiple memory banks in order to be satisfied, via a reduction from the $k$-densest subgraph problem, it is $N P$-complete to determine whether there exists a schedule satisfying $C$ jobs and being active for at most $A$ units of time.

${ }^{*}$ Dept. of Computer Science and Engineering, University of Washington, Seattle WA 98195, jschang@cs.washington.edu. Research supported by an NSF Graduate Research Fellowship and NSF CCF-1016509.

${ }^{\dagger}$ University of Colorado, Boulder CO 80309, hal@cs.colorado.edu.

${ }^{\ddagger}$ Dept. of Computer Science, University of Maryland, College Park MD 20742, samir@cs.umd.edu. Research supported by NSF CCF-0728839, NSF CCF-0937865 and a Google Research Award. 
We propose a simple model for measuring energy usage on a parallel machine. Rather than focusing on conventional metrics measuring the quality of the schedule, we focus on problems motivated by energy savings in "efficient" schedules.

In many applications, a job has many intervals of availability because, e.g., it interfaces with an external event such as a satellite reading or a recurring broadcast. The real-time and period scheduling literatures address problems in this space. More broadly, tasks may be constrained by user availability, introducing irregularity in the feasible intervals. Our model is defined generally enough to capture jobs of this nature.

More formally, we are given a collection of $n$ jobs, each job $J_{i}$ having an integer length $\ell_{i}$ and a set $T_{i}$ of time intervals with integer boundaries in which it can be feasibly scheduled. In particular, $T_{i}=\left\{I_{k}^{i}=\left[r_{k}^{i}, d_{k}^{i}\right]\right\}_{k=1}^{m_{i}}$ is a non-empty set of disjoint intervals. Note that if $m_{i}=1$, then we can think of job $J_{i}$ as having a single release time and a single deadline. For ease of notation, we may sometimes refer to job $J_{i}$ as job $i$. In addition, time is divided into unit length timeslots and for a given parallelism parameter $B$, the system (or machine) can schedule up to $B$ jobs in a single timeslot. If the machine schedules any jobs at timeslot $t$, we say that it is "active at $t$ ". The goal is to schedule all jobs, i.e. schedule them within their feasible regions, while minimizing the number of slots during which the machine is active. The machine consumes a fixed amount of energy per active slot. In other words, subject to each job $J_{i}$ being scheduled within its feasible region $T_{i}$, and subject to at most $B$ jobs being scheduled at any time, we would like to minimize the total active time spent scheduling the jobs. Note that there may be instances when there is no feasible schedule for all the jobs. However, this case is easy to detect. Note that for a timeslot significantly large (e.g. on the order of an hour), any overhead cost for starting a memory bank is negligible compared to the energy spent being "on" for that unit of time.

To illustrate this model in other domains, consider the following operational problem. Suppose that a ship can carry up to $B$ cargo containers from one port to another. Jobs have delivery requirements leading to release times and deadlines. Finding an optimal schedule corresponds to the minimum number of times we need to send the ship to deliver all the packages on time. The motivating assumption is that it costs roughly the same to send the ship, regardless of load and that there is an upper bound on the load.

We could also consider this as a basic form of "batch" processing similar to the work initiated by Ikura and Gimple [39]. Their algorithm is designed to minimize completion time for batch processing on a single machine for the special case of agreeable 1 release times and deadlines. Baptiste [6] extended the Ikura and Gimple results to general release times and deadlines and an efficient algorithm was recently given by Condotta et. al.[15]. All of these works focus merely on trying to find a feasible schedule (which then can be used as a subroutine to minimize maximum lateness). However in our problem, in addition we wish to minimize the number of batches.

In the scheduling literature, often problems with unit processing times are trivial since they can be solved using matching techniques. However, in different models which allow for overlap in job satisfaction, e.g. broadcast scheduling [22, 12, 11, the problems often turn out to be $N P$-complete; in fact, several variants of broadcast scheduling have been shown to be $N P$-complete [11. The problem considered in this paper also contains an element of "overlap" since we can schedule up to $B$ jobs in a slot at unit cost and wish to minimize the number of active slots.

For the cases of unit length jobs and those in which jobs can be preempted at integral time points, our scheduling problem can be modeled as a bipartite matching problem in which each node on the left needs to be matched with a node on the right. Each node on the right has a capacity of $B$, and we are interested in minimizing the number of nodes on the right that have

\footnotetext{
${ }^{1}$ When the ordering of jobs by release times is the same as the ordering of jobs by deadlines.
} 
non-zero degree. This problem can easily be shown to be $N P$-hard. Hence it is slightly surprising that for unit length jobs with each $T_{i}$ being a single interval, we can develop a fast algorithm to obtain an optimal solution to the scheduling problem defined above 2 . Our algorithm is an almost greedy scheme, which intuitively abides by a lazy activation principle: schedule jobs in batches of size up to $B$ delaying the batch as long as possible. At each step, we select "filler" jobs (with later deadlines) to fill slots which otherwise would have at least one and less than $B$ jobs, based on an Earliest Deadline First (EDF) strategy. The algorithm as described does not quite work, since we may schedule some jobs using the lazy activation principle and later discover that these jobs should have been scheduled earlier to make space for other jobs with later deadlines. One way to address this problem is to dynamically re-assign jobs to time slots. Our first attempt was based on this idea, but it resulted in a slower algorithm with a more complicated analysis. However, we are able to address this issue by pre-processing the jobs to create a new instance with "adjusted" deadlines, so that at most $B$ jobs have the same deadline. Then, no re-assignment of jobs is required. As we will see, for infeasible instances, this algorithm has the additional property that it will schedule the maximum number of jobs.

In particular, as our paper shows, even the problem where $B=2$ has a lot of structure due to its connection with matchings in graphs. We anticipate that this structure will be useful in the design of improved approximation algorithms for $B>2(\operatorname{an} O(\log n)$ approximation algorithm follows from the work of Wolsey [57]).

\section{Main Results:}

1. For the case where jobs have unit length and each $T_{i}$ is a single interval, we first develop an algorithm whose running time is $O(n \log n)$. We then show how to improve its running time to linear. Our algorithm takes $n$ jobs as input with integral release times and deadlines and outputs a schedule with the smallest number of active slots. The algorithm has the additional property that for infeasible instances, it schedules the maximum number of jobs. We also note that the slotted aspect of the time model is but a technical convenience. It can be shown without loss of generality that time is slotted when job lengths, release times and deadlines are integral (Section 2).

2. When the release times and deadlines are not integral, non-preemptively scheduling unit length jobs to minimize the number of batches can be solved optimally in polynomial time via dynamic programming (Section 3 ). This objective differs from active time: a batch must start all its jobs at the same time and the system may work on at most one batch at a time. Even so, scheduling unit length jobs with integral release times and deadlines to minimize active time is clearly a special case of this. We extend the result to the case when we have a budget on the number of active slots (Section 4).

3. In addition, we consider the generalization to arbitrary $T_{i}$. This problem is closely related to vertex cover with hard capacities, the $k$-center problem and capacitated facility location, all classic covering problems. In particular, for the special case where every job is feasible in exactly two timeslots, there is a LP-rounding 2-approximation, which is implied from the vertex cover result in [29]. The complexity of the problem depends on the value of $B$, since for any fixed $B \geq 3$, the problem is $N P$-hard. When $B=2$ this problem can be solved optimally in $O(m \sqrt{n})$ time where $m$ is the total number of time slots which are feasible for some job (Section 5). We show that this problem is essentially equivalent to the maximum matching

\footnotetext{
${ }^{2}$ The problem can be solved in $O\left(n^{2} T^{2}(n+T)\right.$ time using Dynamic Programming as was shown by Even et. al. 21, albeit the complexity of their solution is high. Their algorithm solves the problem of stabbing a collection of horizontal intervals with the smallest number of vertical stabbers, each stabber having a bounded capacity.
} 
problem computationally. In addition, we show that this algorithm can be extended to the case of non-unit length jobs when a job can be scheduled in unit sized pieces (Section 6).

4. We also consider a version of the problem when jobs have arbitrary lengths and can be preempted at any point in time, i.e. not just at integer time points. For general $B$ the problem can be solved by linear programming. For $B=2$ the problem amounts to finding a maximum triangle-free 2-matching on a special graph. Babenko et. al. present an elegant algorithm showing that a maximum cardinality triangle-free 2-matching can be found in the same time as a maximum cardinality matching [5]. We extend it for our scheduling problem to show that when $B=2$ and jobs have arbitrary integral length, an optimal preemptive schedule can be found in $O(\sqrt{L} m)$ time, for $L$ the total length of all jobs. Any preemptions occur at integral or half-integral times.

5. In Section 7, we also give a tight bound on the gain from arbitrary preemption: an optimal schedule allowing only preemption at integral times uses at most $4 / 3$ the active time of the optimal preemptive schedule. We also note that this bound is best possible since there is a trivial example with three unit jobs where the optimal schedule which allows preemption only at integer points uses two slots, and if we allow arbitrary preemptions, these jobs can be scheduled in 1.5 slots giving the ratio of $4 / 3$.

\subsection{Related Work}

A classical problem related to our work is the well known "Scheduling unit jobs on $B$ processors with precedence constraints", in which $n$ unit jobs are given with precedence constraints and the goal is to schedule the jobs on $B$ processors to minimize the maximum completion time. Again this can be viewed as minimizing the active time. For arbitrary $B$ the problem is $N P$-complete [34]. For fixed $B$, the problem is known to be $\mathrm{W}[2]$ hard [9]. For the case where $B=2$, this problem can be solved optimally in polynomial time [25, 28]. Garey and Johnson [35, 36] consider the problem of scheduling unit jobs with integer release times and deadlines with precedence constraints, providing polynomial time algorithms for $B=2$. In addition, their algorithm finds a schedule with minimum lateness. This was extended to the case of real release times and deadlines by Wu and Jaffar [58], who gave an $O\left(n^{4}\right)$ algorithm. Their primary technique involves computing successor-tree-consistent deadlines, which effectively upper bounds the latest completion time for each job. Successor-tree-consistency allows the optimal schedule to be computed via a slight variation to Simon's forward scheduling algorithm for independent unit length jobs. For scheduling unit length jobs with arbitrary release times and deadlines on $B$ processors to minimize the sum of completion times, Simons and Warmuth [55] extended the work by Simons [54] giving an algorithm with running time $O\left(n^{2} B\right)$ to find a feasible solution. For constant $B$, the running time is improved in [48].

A closely related problem of minimizing busy time has been recently studied by Khandekar, Schieber, Shachnai and Tamir [44. In the busy time problem, jobs of arbitrary length have individual demands $r_{j}$. The jobs have release times and deadlines and need to be scheduled in batches, with the additional requirement that the total demand of jobs in the batch at any point of time is at most a given value. Each batch is scheduled on a single machine. The busy time of a batch is defined as the busy time of the machine that schedules it, i.e. the duration of the earliest start time to latest end time of jobs in the batch. We highlight that their model permits access to an unbounded number of machines; thus every instance is feasible, albeit with potentially high cost. Our problem is slightly different in that we have uniform demands (as in [23]), and we do not have an unbounded number of machines. The non-unit length generalization makes the problem $N P$-hard, even for the uniform demand case [23, 56]. In [23, the authors consider the uniform 
demand case and present a 4-approximation as well as results for the special case where the jobs are interval jobs, i.e. the processing time is exactly the length of the interval. In [44], they consider a more general problem and develop an approximation algorithm with a factor of 5 . Their main idea is to first "cluster" the jobs with the assumption that each batch has infinite capacity and then fix this as the position of the job by modifying the release time and deadline, thus converting it to an interval job. The main algorithm then partitions the jobs by demand into two categories and uses a greedy method to schedule the jobs. A number of applications are mentioned in [44, 23.

Baptiste [7] examines a related problem of "min gap" scheduling unit length jobs on a single processor to minimize the number of idle intervals; in this model, the algorithm determines when the processor sleeps. They give an optimal dynamic programming algorithm which builds from a dominance property of the optimal offline schedule. Baptiste, Chrobak and Dürr in [8] improve the running time; their algorithm in fact applies to the generalized problem in which jobs have arbitrary processing times. This work was subsequently extended to handle multiple processors by Demaine et. al. [18, who also provide an approximation algorithm for the case where each job has multiple intervals in which it can be scheduled. They also give $\log n$ lower bounds on the approximation ratio. The cost function in this lower bound does not apply to the problems studied in this paper.

Also related is the dynamic speed scaling problem, in which the scheduler determines the nonnegative speed at which the processor runs. For a single processor, Yao et al. 59 give an exact offline solution that minimizes the total power consumption when the power is a convex function of the speed. Irani et al. [40] study an extended problem in which the machine can also be put into the "sleep" state, during which period no cost is incurred other than the constant wake-up cost. They present a 2-approximation and an $O(1)$-competitive algorithm in the online setting. The problem was recently shown to be $N P$-complete and the approximation improved to $4 / 3$ by Albers and Antoniadis [3]. Despite the significant results in [40, its authors acknowledge that a continuous power function is unrealistic; in practice, systems run at a finite number of potential speeds. Our work is the special case in which power is represented by a step function.

$\mathrm{Li}$ and Yao [47] consider a discretized version of the problem, in which the system may operate at one of a finite number of speeds. Their algorithm is exact and runs in time $O(d n \log n)$, where $d$ is the number of possible speeds. The main idea is to first partition the jobs, and then to determine the speeds of these jobs, partition by partition. However, their model is not quite the same as ours; despite the discretization of the speeds, they still assume that the underlying power function is convex and therefore cannot capture the step from speed 0 to positive speed.

Demaine et. al. [18] investigated problems involving multiple processors and more generally, multiple feasible intervals for jobs. For the multiple processors settings, they provide a polynomialtime algorithm which minimizes the total number of gaps in the schedule. The algorithm also minimizes the total transition energy plus total time in active slots, over multiple processors. Notice that this setting is not quite a generalization of ours, since the total active time is summed over each processor. Unlike our cost model, it is cheaper to activate fewer rather than more processors at any given time. Finally, they give for the multi-interval setting a $\left(1+\left(\frac{2}{3}+\epsilon\right) \alpha\right)$-approximation, where $\alpha$ is the cost to transition to the active state. Notice that their cost model is very closely related to ours, with the exception that we consider settings in which the startup cost is negligible enough to be assumed zero. Thus, their lower bounds on the approximation ratio, which explicitly assume a non-zero $\alpha$, do not apply to our problem.

Demaine and Zadimoghaddam [19] recently studied the problem of minimizing energy consumption in schedules over multiple processors. Their model is quite general in that the feasible time slots in which each unit length job may be scheduled may not comprise a single time interval. Also, each processor has an arbitrary (unrelated) energy function and can (but doesn't have to) go to 
a sleep state. They provide a $O(\log n)$-approximation for this problem by first proving a general result for the submodular maximization problem with budget constraints, and then reducing their scheduling problem to a matching problem on a bipartite graph with a submodular matching function. They also show that the problem is Set-Cover-hard, thus demonstrating the tightness of their result.

We refer the reader to surveys [41, 1, 2] for a more comprehensive overview of the latest scheduling results for power management problems.

\section{Lazy Activation for Unit Jobs and Single Execution Windows}

We first provide a high level description of the algorithm, followed by pseudo-code and the proof of optimality. We may assume that the instance is feasible, since this is easy to check by an EDF computation.

Denote the distinct deadlines by $d_{i_{1}}<d_{i_{2}}<\ldots<d_{i_{k}}$, and let $S_{p}$ be the set of jobs with deadline $d_{i_{p}}$. Then $S_{1} \cup S_{2} \ldots \cup S_{k}$ is the entire set of jobs where the deadlines range from time 1 to $T=d_{i_{k}}$. It is not hard to argue that w.l.o.g. $T$ is $O(n)$.

We process the jobs in two phases. In Phase I we scan the jobs in order of decreasing deadline. We do not schedule any jobs, but only modify the deadlines of jobs to create a new instance, whose optimal solution is equivalent to that of the original instance. The desired property of the new instance is that at most $B$ jobs will have the same deadline. Process the time slots from right to left. At slot $D$, let $S$ be the set of jobs that currently have deadline $D$. From $S$, select $\max (0,|S|-B)$ jobs with earliest release times and decrement their deadlines by one. If $|S| \leq B$ then we do not modify the deadlines of jobs in $S$. (Note that a job may have its deadline reduced multiple times since it may be processed repeatedly.)

Assume for simplicity's sake that after the first phase, $S_{p}$ refers to the jobs of (modified) deadline $d_{i_{p}}$. We now describe Phase II in which jobs are actually scheduled. Initially all jobs are unscheduled. As the algorithm assigns jobs to active time slots, we change the status of jobs to scheduled. Once a job is scheduled, it remains scheduled. Once a slot is declared active, it will remain active for the entire duration of the algorithm.

Our algorithm, in general, schedules jobs in increasing order by deadline. As we will see shortly, this is not quite true since we may schedule some jobs with later deadlines whenever there is available space. In precise terms, we schedule the time slots $d_{i_{p}}$ from left to right. To schedule $d_{i_{p}}$, if there are still unscheduled jobs with that deadline we schedule them. If fewer than $B$ jobs get scheduled, we schedule additional jobs that are available. Job $j$ is available if it is currently unscheduled and has $d_{i_{p}} \in\left[r_{j}, d_{j}\right)$. We schedule these available jobs EDF, until the slot is full or no more jobs are available.

\subsection{Formal Algorithm Description}

Let $B>0$ be the number of jobs that the system can satisfy in a single time slot. For every job $j$, denote $j$ 's release time and deadline by $r_{j}$ and $d_{j}$, respectively. We index the $n$ jobs in order of increasing deadline, i.e. such that $d_{1} \leq d_{2} \leq \ldots \leq d_{n}=T$. Normalize to 0 the earliest release time of any job. Then without loss, all feasible schedules are active only within the interval $[0, T]$.

In the first phase we scan the jobs from right to left in decreasing deadline order. At each step we consider the set of jobs with a common deadline and leave up to $B$ jobs with the latest release times untouched. We modify the deadlines of the rest of the jobs in this set, decrementing them each by one, and then continue processing the jobs. 
The algorithm for the second phase simultaneously maintains a set $W$ of active time slots and a set $J$ of satisfied jobs, both of which are initially empty. In each iteration, we look at the unsatisfied job $j^{*}$ of earliest deadline, i.e. $j^{*}=\arg \min _{j \notin J} d_{j}$. Let $d^{*}$ be $j^{*}$ s deadline, and let $J^{*}$ be the set of all unsatisfied jobs with the same deadline $d^{*}$. The algorithm activates the latest possible time slot which can satisfy $J^{*}$ and adds it to $W$. Only one slot is needed to satisfy $J^{*}$ since $\left|J^{*}\right| \leq B$.

The algorithm assigns jobs to the newly activated time slot $t$ first adding $J^{*}$ to $J$ and assigning those jobs to slot $t$. If fewer than $B$ jobs are available, we fill the remaining space by selecting available (filler) jobs from the remaining set of unscheduled jobs, again selecting based on EDF. These filler jobs will then be added to $J$.

The following pseudocode formalizes the above description of the second phase. SelectFillers takes as input the set of active slots $W$ and the set of scheduled jobs $J$, returning the set of filler jobs $J^{\prime}$. These jobs are then added to the set of scheduled jobs.

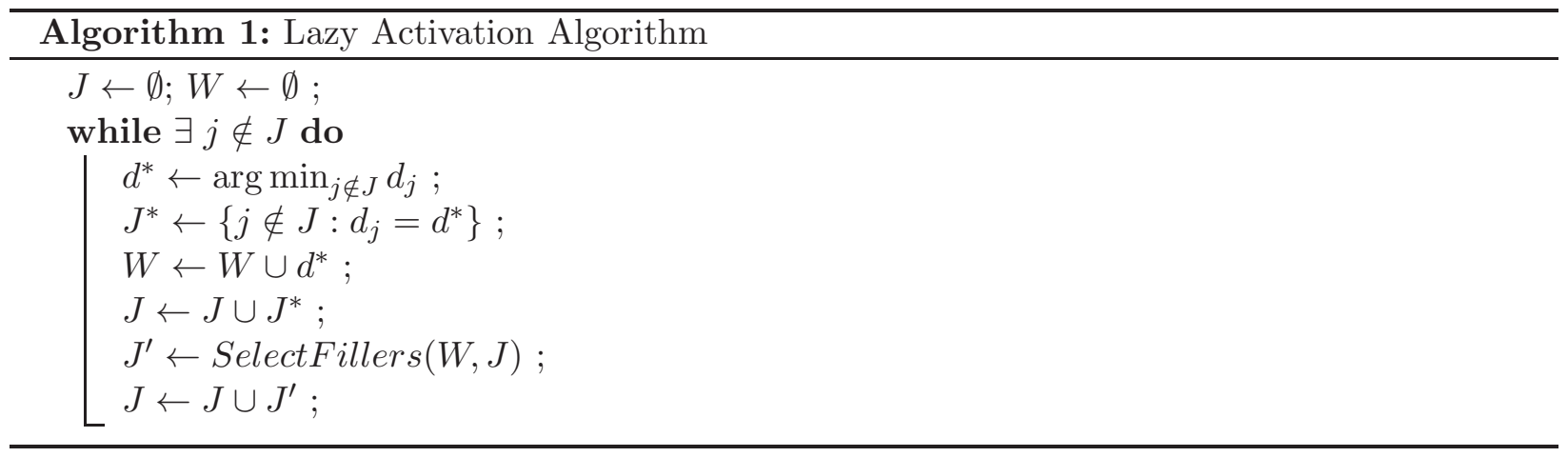

\begin{tabular}{c} 
Algorithm 2: SelectFillers $(W, J)$ \\
Choose available fillers based on EDF to fill the $B-\left|J^{*}\right|$ empty spots. \\
\hline
\end{tabular}

\subsection{Analysis of the Algorithm}

It is easy to implement our algorithm in running time $O(n \log n)$ using standard data structures. What is not completely obvious is why it computes an optimal solution.

Suppose the initial instance $I$ is transformed by Phase $I$ to a modified instance $I^{\prime}$. We prove the following properties about an optimal solution for $I^{\prime}$.

Proposition 1 An optimal solution for $I^{\prime}$ has the same number of active slots as an optimal solution for the original instance $I$.

Proof: It is easy to see that any feasible solution for $I^{\prime}$ is feasible for $I$ since pre-processing only created a more constrained instance in the transformation; each job's window in $I^{\prime}$ is a subset of its window in the original instance $I$. We now argue that a solution for $I$ can be transformed to a solution for $I^{\prime}$ using the same number of slots. Suppose a feasible schedule $\sigma$ for $I$ is infeasible for $I^{\prime}$ due to a job $x$. In other words, $\sigma$ schedules job $x$ after its modified deadline in $I^{\prime}$. We can argue this step by step, by showing that decrementing the deadline of a single job does not change the optimal solution; since the modification is done by a sequence of such operations, the optimal solution is preserved. Assume that the deadline of $x$ was reduced by one. In the instance $I^{\prime}$, we have $B$ jobs with deadline $d_{x}$, out of which at most $B-1$ jobs can be scheduled with $x$. Hence 
there is at least one job scheduled earlier whose deadline is still $d_{x}$. Since its release time cannot be before the release time of $x$, we can exchange these two jobs. This makes the schedule feasible for $I^{\prime}$, and this establishes the proposition.

Proposition 2 Without loss of generality an optimal solution for $I^{\prime}$ uses a subset of slots that are deadlines.

Proof: Among all optimal solutions select the one that uses the least number of non-deadline slots. Among all the slots that are not deadlines, choose the slot $t$ that is the right-most (i.e. latest) active slot. Let $X$ be the set of jobs which are assigned to $t$. We now move $X$ as late as possible while maintaining feasibility for every job in $X$. We will end up next to a deadline slot $t^{\prime}$ containing a set of blocking jobs $B$; otherwise we will have reduced the number of non-deadline slots. From the set of jobs $X \cup B$, select $B$ jobs with the earliest deadlines and schedule them in $t^{\prime}$. Since at most $B$ jobs have deadline $t^{\prime}$, the remaining jobs all have higher deadlines and the process can be repeated.

Theorem 3 Algorithm Lazy Activation computes an optimal solution for $I^{\prime}$ (with the smallest number of active slots).

Proof: It is enough to prove that the optimal solution w.l.o.g. schedules exactly the same jobs that we schedule in the first active slot (earliest deadline $d_{1}$ ). By removing these jobs from the instance $I^{\prime}$, it is easy to see that our algorithm computes an optimal solution. The proof for the claim is as follows. Due to the above propositions, the jobs with deadline $d_{1}$ w.l.o.g. are all scheduled in time slot $d_{1}$ in the optimal solution; Algorithm Lazy Activation also schedules all the jobs in this time slot. In addition, observe that to fill the remaining slots among the available slots, the optimal solution again w.l.o.g. selects available jobs with earliest deadline, i.e. the filler jobs chosen by Algorithm Lazy Activation. Otherwise we can exchange the jobs to achieve this property. The proof follows by induction.

\subsection{On Infeasible Instances}

In this section, we consider the behavior of the Lazy Activation algorithm on instances for which it is impossible to schedule all jobs within their individual windows of feasibility. We will show that Lazy Activation maximizes the number of jobs satisfied. In fact, we will see that it does so in the fewest number of active timeslots. Denote by $\mathcal{S}_{L A}$ the schedule returned by Lazy Activation. In Phase I, it is possible for a job's deadline to be decremented all the way to its release time; in this case, we say that the job's window has collapsed.

Proposition 4 On infeasible instances, Lazy Activation maximizes the number of jobs satisfied.

Proof: One can use an argument similar to Proposition 1 to show that even if Phase I collapses the windows of some jobs, it does not change the maximum number of jobs that can be scheduled. For completeness' sake, we detail it here. As before, we argue step-by-step that each decrement changing the instance from $I$ to $I^{\prime}$ does not affect the maximum throughput. Suppose a deadline $d_{x}$ of job $x$ is reduced by one. Let $\sigma$ be a feasible schedule on $I$ achieving maximum throughput on $I$. We can transform $\sigma$ into $\sigma^{\prime}$ that is feasible on instance $I^{\prime}$ and that satisfies the same number of jobs. If $\sigma$ does not schedule job $x$ at $d_{x}$, then $\sigma$ is already feasible on $I^{\prime}$. Suppose $\sigma$ schedules $x$ at $d_{x}$. Then $\sigma$ can do at most $B-1$ other jobs at $d_{x}$. In $I^{\prime}$, there are $B$ jobs with deadline $d_{x}$ 
and release time $\geq r_{x}$. Thus, there exists a job $j$ which is not scheduled by $\sigma$ at $d_{x}$ and which has release time at least $r_{x}$. Modify $\sigma$ by swapping jobs $j$ and $x$. (If $j$ was not scheduled in $\sigma$, then schedule $j$ and not $x$.) The new schedule satisfies the same number of jobs and is also feasible for $I^{\prime}$.

Thus, the modification of deadlines in Phase I does not change the maximum number of jobs which can be scheduled. In particular, jobs whose windows collapse in Phase I w.l.o.g. are also dropped in some throughput-maximizing schedule. In Phase II, Lazy Activation schedules every job whose window has not collapse, either at its deadline or earlier, i.e. as a filler. Therefore, Lazy Activation maximizes the number of jobs satisfied.

Proposition 5 If Lazy Activation's schedule $\mathcal{S}_{L A}$ satisfies $n^{\prime}<n$ jobs in $k$ active slots, then any schedule $\mathcal{S}$ satisfying $n^{\prime}$ jobs does so in at least $k$ active time slots.

Proof: Suppose that Lazy Activation collapses $\kappa=n-n^{\prime}$ jobs, denoted $j_{a_{1}} \ldots j_{a_{\kappa}}$. Let $\alpha_{t}$ be the number of jobs that had deadline $t$ at the start of the iteration in which Phase I processed $t$ as the deadline. Deadlines will be decremented precisely when $\alpha_{t}>B$. For each collapsed job $j_{a_{i}}$, we will identify an interval $I_{a_{i}}$ of excess demand by intuitively "unrolling" the iterations of Phase I starting from the point of collapse. More formally, set $t^{\prime}$ to the latest slot such that every slot $t \in\left[r_{j}, t^{\prime}\right]$ is such that $\alpha_{t}>B$. Define $I_{a_{i}}$ to be $\left[r_{j}, t^{\prime}\right)$. Notice that $t^{\prime}$ is the original deadline of some job. Let $J_{a_{i}}=\left\{j:\left[r_{j}, d_{j}\right) \subseteq I_{a_{i}}\right\}$. Then the collapsed job $j_{a_{i}} \in J_{a_{i}}$ and also $\left|J_{a_{i}}\right|>B \cdot\left|I_{a_{i}}\right|$. In fact, $J_{a_{i}}$ consists exactly of two types of jobs: jobs which have collapsed and $B \cdot\left|I_{a_{i}}\right|$ jobs which have not collapsed. Lazy Activation schedules the latter job set in $I_{a_{i}}$ at $B$ jobs per slot.

Now partition the original instance $(J, T)$ into two subinstances $\left(J_{1}, T_{1}\right)$ and $\left(J_{2}, T_{2}\right)$, where $J_{1}=\bigcup_{i=1}^{k} J_{a_{i}}$ and $T_{1}=\bigcup_{i=1}^{k} I_{a_{i}}$. Obviously jobs of $J_{1}$ cannot be scheduled in slots of $T_{2}$ by definition. We claim that since $\mathcal{S}$ maximizes throughput, it necessarily schedules $J_{2}$ in $T_{2}$. Suppose there exists a job $j \in J_{2}$ that is scheduled by $\mathcal{S}$ in some interval $I_{a_{i}}$. Then since $\left|J_{a_{i}}\right|>B \cdot\left|I_{a_{i}}\right|$, there is some job of $J_{a_{i}}$ that is missed by $\mathcal{S}$. Since it is possible to schedule all jobs of $J_{2}$ only in slots of $T_{2}$ ( $\mathcal{S}_{L A}$ is such an example), missing that many jobs of $J_{a_{i}}$ was unnecessary. This contradicts the fact that $\mathcal{S}$ maximizes throughput.

Then the active time $A(\mathcal{S})$ of $\mathcal{S}\left(\mathcal{S}_{L A}\right.$, respectively) can be decomposed into two components: the active time $A_{1}(\mathcal{S})$ spent satisfying $J_{1}$ and the active time spent satisfying $J_{2}$. Then,

$$
\begin{aligned}
A(\mathcal{S}) & =A_{1}(\mathcal{S})+A_{2}(\mathcal{S}) \\
& \geq A_{1}\left(\mathcal{S}_{L A}\right)+A_{2}\left(\mathcal{S}_{L A}\right) \\
& =A\left(\mathcal{S}_{L A}\right) \\
& =k
\end{aligned}
$$

where the inequality follows from the facts that (1) Lazy Activation minimizes active time on feasible instances and (2) there are exactly $B \cdot\left|I_{a_{i}}\right|$ jobs contained in each $I_{a_{i}}$ that have not collapsed and Lazy Activation schedules all of them. Thus whenever Lazy Activation is active in $T_{2}$, it schedules $B$ jobs per slot.

\subsection{Linear Time Implementation}

We conclude by showing that the algorithm can be implemented in time $O(n+T)$. We start by giving the following equivalent version of Phase I, which we refer to as Phase $I^{\prime}$. Note that in "assigning" jobs to deadlines, both Phases are only preprocessing deadlines. The actual schedule is not developed until Phase II. 
Initially each new deadline value has no jobs assigned to it. Process the jobs $j$ in order of decreasing release time $r_{j}$ (jobs with the same release time can be processed in arbitrary order). Assign $j$ to a new deadline equal to the largest value less than or equal to $d_{j}$ that currently has $<B$ jobs assigned to it.

To prove that Phase $\mathrm{I}^{\prime}$ computes the same deadlines as Phase I, assume that both algorithms break ties for release times the same way. Let $D_{j}$ and $D_{j}^{\prime}$ denote the deadlines to which job $j$ is assigned by Phase I and Phase $\mathrm{I}^{\prime}$, respectively. We show that for all $j, D_{j}^{\prime}$ is equal to $D_{j}$, by induction on Phase I's iterations. Clearly this holds for the first iteration, since the first job considered has maximum release time, so Phase I never decrements its deadline. Therefore, both Phases assign it to its original deadline. Now consider some job $j$ and suppose that equality holds for all jobs processed it iterations previous to that of job $j$. Then $D_{j} \leq D_{j}^{\prime}$ : $D_{j}$ is an available candidate deadline for Phase I', which chooses the latest such one. Thus, when Phase I assigns jobs to deadline $D_{j}^{\prime}$, it considers assigning job $j$ (feasible and unassigned) there as well. In fact, Phase I assigns to $D_{j}^{\prime}$ the jobs that Phase $\mathrm{I}^{\prime}$ has already assigned there. There are less than $B$ of them, each having release time at least $r_{j}$. Among the remaining jobs which are feasible at $D_{j}^{\prime}$, job $j$ has the maximum release time so Phase I assigns $j$ to $D_{j}^{\prime}$. Therefore, $D_{j}=D_{j}^{\prime}$, as claimed.

We implement Phase $\mathrm{I}^{\prime}$ using an algorithm for disjoint set merging on the universe of possible deadline values $1, \ldots, T$. Each set consists of a deadline $D$ to which $<B$ jobs have been assigned, plus the maximal set of consecutive values $D+1, \ldots$ that each have $B$ assigned jobs. It is easy to see that using the set merging data structure of [33] achieves time $O(n+T)$ for Phase $\mathrm{I}^{\prime}$.

Phase II constructs an earliest-deadline first schedule on the timeslots that are Phase I deadlines. In particular, let $D_{1}<D_{2}<\ldots<D_{\ell}$ be the distinct deadline values assigned to jobs in Phase I. Modify release times and deadlines to compress the active interval from $[0, T]$ to $[0, \ell]$, as follows: a job $j$ with Phase I deadline $D_{k}$ gets deadline $k$; its release time $r_{j}$ gets changed to the largest integer $i$ such that $D_{i} \leq r_{j}$ (take $\left.D_{0}=0\right)$. The algorithm computes an earliest-deadline first schedule on this new instance. It returns the corresponding decompressed schedule, with slot $i$ changed back to $D_{i}$.

Phase II begins by assigning the new release times in time $O(n+T)$. Then it constructs an EDF schedule using disjoint set merging as in Phase $\mathrm{I}^{\prime}$ [24], achieving time $O(n+T)$ [33].

\subsection{Slotted versus Unslotted Model}

Here we show that even if the time model is not slotted, the fact that the release times and deadlines are integral implies that without loss, the optimal solution schedules jobs in slots implied by integer time boundaries.

Theorem 6 Without loss of generality, the optimal solution schedules jobs so that they start and end at integral time points.

Proof: Call a job non-integral if it starts (and ends) at a non-integral time. Suppose an optimal solution $\mathcal{O}$ has non-integral jobs. We modify $\mathcal{O}$ to another optimal schedule $\mathcal{M}$ with fewer such jobs. Doing this repeatedly eliminates all non-integral jobs.

Let $I$ be the total inactive time of $\mathcal{O}$ 's schedule, i.e., an optimal schedule maximizes $I$. In $\mathcal{O}$, let $[t, t+1$ ) be the last interval (for $t$ an integer) in which a non-integral job ends.

Case 1: Some processor is busy throughout the interval $[t, t+1)$ (e.g., for unit jobs this means some job starts at $t$ ). Construct $\mathcal{M}$ by delaying every non-integral job which ends in this slot so that it ends at $t+1$ instead. $I$ does not decrease, since every job gets moved into a time period 
that was already active in $\mathcal{O}$. Clearly, the number of non-integral jobs decreases.

Case 2: No processor is busy during the entire interval $[t, t+1)$. Let $i$ be the largest integer $<t$ such that an interval of positive length $\left[i, i^{\prime}\right)$ is inactive $\left(i^{\prime}>i\right)$. If no such interval exists, let $i=0$.

Construct $\mathcal{M}$ by taking every non-integral job that starts after $i$ and advancing its start time by $\epsilon=\min \{s-\lfloor s\rfloor: s>i$ the starting time of a non-integral job $\}$. Obviously $0<\epsilon<1$. Observe that the definition of $i$ implies than a non-integral jobs advances if and only if it ends after $i$.

$\mathcal{M}$ is a valid schedule: the choice of $\epsilon$ guarantees that no release time is violated. We claim that no processor executes more than one job at any point in time, i.e. no job $j$ advances into the execution of a job $j^{\prime}$ that does not advance. In proof, if $j^{\prime}$ is integral, then it ends at an integral time. The definition of $\epsilon$ shows that $j$ does not advance past an integral time. If $j^{\prime}$ is non-integral, then it ends at or before $i$. Again, the definition of $\epsilon$ implies that $j$ does not advance past integer time $i$.

To show that $\mathcal{M}$ is optimal, we will show that $I$ does not change. Specifically, we show that $I$ increases by $\epsilon$ at the end of the schedule, $I$ does not change in the middle, and $I$ decreases by $\epsilon$ at the start.

In interval $[t, t+1$ ), every job advances by $\epsilon$ (by Case 2), so $I$ increases by $\epsilon$ in this interval.

Next, consider a maximal interval $[a, b)$ that is inactive in $\mathcal{O}$ and starts at $a>i$. We claim that $\mathcal{M}$ is inactive during a corresponding interval $\left[a^{\prime}, b^{\prime}\right)$ of the same or greater length. The definition of $i$ implies that $a$ is non-integral, i.e., $a$ is the ending time of a non-integral job. This ending time moves to $a^{\prime}=a-\epsilon$. If $b$ is the start time of a non-integral job, that start moves to $b^{\prime}=b-\epsilon$. Clearly, $\left[a^{\prime}, b^{\prime}\right)$ is inactive in $\mathcal{M}$. The other possibility is that $b$ starts an integral job. Setting $b^{\prime}=b$ gives an inactive interval of greater length.

If $i=0$, we have shown that $\mathcal{M}$ has more inactive time than $\mathcal{O}$, a contradiction. If $i>0$, the interval $\left[i, i^{\prime}\right)$ shrinks to an interval of inactive time $\left[i, i^{\prime}-\epsilon\right)$ in $\mathcal{M}$. This decreases $I$ by $\epsilon$. We conclude that $\mathcal{M}$ is an optimal schedule. The definition of $\epsilon$ implies that $\mathcal{M}$ has more integral jobs than $\mathcal{O}$.

\section{Unit Jobs with Arbitrary Release Times and Deadlines}

In this section, we consider a generalization of the previously discussed problem. Suppose that the unit length jobs have release times and deadlines over the reals and that we want to minimize the number of batches in the schedule, where a batch is a set of jobs which all start and finish at the same time and where the system can work on at most one batch at a time. This objective is slightly more restrictive than active time; even so, scheduling unit length jobs with integer release times and deadlines to minimize active time is clearly a special case of this, since in the former problem, the batch property holds without loss.

We describe a simple dynamic program which determines in $O\left(n^{8}\right)$ time the minimum number of batches needed to non-preemptively satisfy all jobs, i.e. $1 \mid p$-batch, $B<n, r_{i}, d_{i}, p_{i}=1 \mid K$, where $K$ is the number of batches in the schedule. The dynamic program (and therefore, the notation) is similar to that found in [6], on which several DP results in this area of scheduling are based. Suppose throughout that jobs are listed in ascending order by deadline, i.e. $d_{1} \leq d_{2} \leq \ldots \leq d_{n}$.

Definition 1 For job $k$ and for interval $\left[t_{\ell}, t_{r}\right]$ where $r_{k} \in\left[t_{\ell}, t_{r}\right]$ and $t_{r}+1 \leq d_{k}$, let the set of jobs $U_{k}\left(t_{\ell}, t_{r}\right)=\left\{j \leq k: r_{j} \in\left[t_{\ell}, t_{r}\right]\right\}$ (see Fig. 11). Also let $U_{0}\left(t_{\ell}, t_{r}\right)=\emptyset$ for all intervals $\left[t_{\ell}, t_{r}\right]$. 


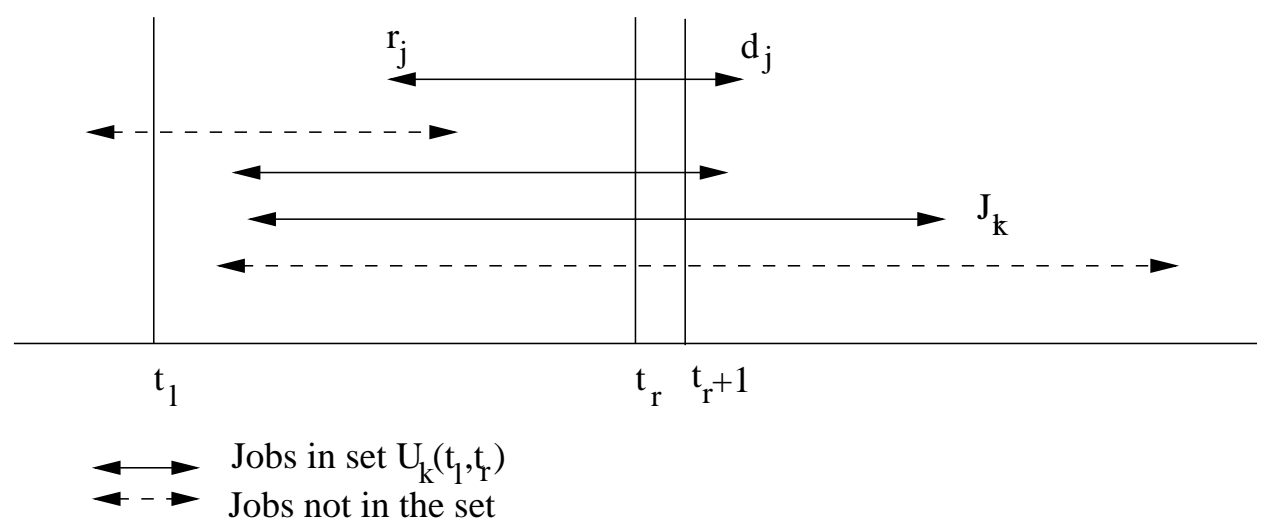

Figure 1: Jobs of $U_{k}\left(t_{\ell}, t_{r}\right)$.

Observe that the jobs in $U_{k}\left(t_{\ell}, t_{r}\right)$ must be scheduled entirely in $\left[t_{\ell}, d_{k}\right]$ in any feasible schedule. We restrict ourselves without loss to the space of schedules which obey the EDF Principle: for any pair of jobs $i$ and $j$ where $i<j$, either job $i$ is scheduled before or with job $j$, or $r_{i}$ is after the point at which job $j$ is scheduled. Indeed, if a schedule contains a pair of jobs $i$ and $j$ for which this does not hold, then one can swap them in the schedule without affecting feasibility or the number of batches.

Definition 2 Let $P\left(k, t_{\ell}, t_{r}, \mu_{r}\right)$ be the minimum number of batches required to schedule $U_{k}\left(t_{\ell}, t_{r}\right)$ such that (1) all batches start within $\left[t_{\ell}+1, t_{r}\right]$ and (2) at most $B-\mu_{r}$ of these jobs are scheduled in the batch starting at $t_{r}$, and if $\mu_{r}>0$, this coming at no additional cost.

If such a schedule does not exist, e.g. $k>0$ and $t_{\ell}+1>t_{r}$, then let $P\left(k, t_{\ell}, t_{r}, \mu_{r}\right)=\infty$. Let $P_{t}\left(k, t_{\ell}, t_{r}, \mu_{r}\right)$ be the value of $P\left(k, t_{\ell}, t_{r}, \mu_{r}\right)$ subject to job $k$ being scheduled in a batch that starts at time $t$. Then

$$
P\left(k, t_{\ell}, t_{r}, \mu_{r}\right)=\min _{t} P_{t}\left(k, t_{\ell}, t_{r}, \mu_{r}\right)
$$

Per Baptiste's observation in [7, it is enough to iterate over a set of $O\left(n^{2}\right)$ possible starting times for batches. For a given $t$, one can compute $P_{t}\left(k, t_{\ell}, t_{r}, \mu_{r}\right)$ as follows. Let $L=\left\{j \leq k: r_{j} \in\left[t_{\ell}, t\right]\right\}$ be the jobs of $U_{k}\left(t_{\ell}, t_{r}\right)$ which are released before or at $t$. Similarly, let $R=\left\{j \leq k: r_{j} \in\left(t, t_{r}\right]\right\}$ be the jobs of $U_{k}\left(t_{\ell}, t_{r}\right)$ released after $t$. The observation above implies that if $i \in L$, then $i$ is scheduled before or at $t$. On the other hand, if $i \in R$, then job $i$ must be scheduled after $t$. Let $k_{L} \in \arg \max _{i \in L \backslash\{k\}} d_{i}$, or 0 if $L \backslash\{k\}=\emptyset$. Similarly, let $k_{R} \in \arg \max _{i \in R} d_{i}$, or 0 if $R=\emptyset$.

If $t=t_{r}$, then $L=U_{k}\left(t_{\ell}, t_{r}\right), R$ is empty and $k_{L}$ (if positive) is the second-to-latest deadline in $U_{k}\left(t_{\ell}, t_{r}\right)$. Then,

$$
P_{t}\left(k, t_{\ell}, t_{r}, \mu_{r}\right)= \begin{cases}P\left(k_{L}, t_{\ell}, t_{r}, \mu_{r}+1\right) & \text { if } 0<\mu_{r}<B \\ 1+P\left(k_{L}, t_{\ell}, t_{r}, 1\right) & \text { if } \mu_{r}=0 \\ \infty & \text { otherwise }\end{cases}
$$

Consider $t<t_{r}$. If $\mu_{r}>0$, then for $t \in\left(t_{r}-1, t_{r}\right), P_{t}\left(k, t_{\ell}, t_{r}, \mu_{r}\right)=\infty$, since starting batches at $t$ and at $t_{r}$ will violate the constraint that at any given time, there is at most one batch running. Otherwise, i.e. if $t<t_{r}-1$ or $\mu_{r}=0$,

$$
P_{t}\left(k, t_{\ell}, t_{r}, \mu_{r}\right)=1+P\left(k_{L}, t_{\ell}, t, 1\right)+P\left(k_{R}, t, t_{r}, \mu_{r}\right)
$$


Finding the optimal number of batches is equivalent to computing $P\left(n, \min _{i} r_{i}-p, d_{n}-1,0\right)$. Since there are $O(n)$ jobs and $O\left(n^{2}\right)$ interesting times to consider, the total running time is $O\left(n^{7} B\right)=O\left(n^{8}\right)$. The running time was recently improved to $O\left(n^{3}\right)$ in [42].

\section{Maximizing Throughput Subject to Batch Size Constraint}

For infeasible instances, one can apply a technique of the same vein to maximize throughput subject to an upper bound $\mathcal{K}$ on the number of batches. Define $P\left(k, t_{\ell}, t_{r}, \mu_{r}, \kappa\right)$ to be the maximum number of jobs of $U_{k}\left(t_{\ell}, t_{r}\right)$ which can be scheduled in at most $\kappa$ batches starting in $\left[t_{\ell}+1, t_{r}\right]$, where as before, if $\mu_{r}>0$, one can schedule up to $B-\mu_{r}$ jobs in the batch starting at $t_{r}$ without incurring cost toward the batch budget $\kappa$.

To understand the computation of $P\left(k, t_{\ell}, t_{r}, \mu_{r}, \kappa\right)$, let $t$ be the starting time of the batch which satisfies job $k$ (if such a time exists). Consider where the rest of $U_{k}\left(t_{\ell}, t_{r}\right)$ may be satisfied within $\left[t_{\ell}+1, t_{r}\right]$. Denote by $\alpha\left(\beta\right.$, respectively) the number of them which start in $\left[t_{\ell}+1, t\right]\left(\left(t, t_{r}\right]\right.$, respectively). Observe that $\beta \leq \kappa-\alpha$.

There are four cases. Suppose job $k$ is not satisfied in the optimal solution. Then $P\left(k, t_{\ell}, t_{r}, \mu_{r}, \kappa\right)=$ $P\left(k^{\prime}, t_{\ell}, t_{r}, \mu_{r}, \kappa\right)$ where $k^{\prime}=\arg \max _{i \in U_{k}\left(t_{\ell}, t_{r}\right) \backslash\{k\}} d_{i}$, i.e. the job of next latest deadline in $U_{k}\left(t_{\ell}, t_{r}\right)$. (If $U_{k}\left(t_{\ell}, t_{r}\right)=\emptyset$, then $k^{\prime}=0$.) If job $k$ is satisfied in the schedule, then define $L, R, k_{L}$ and $k_{R}$ as in Section 3. Suppose that job $k$ is satisfied in the batch starting at $t=t_{r}$. Then

$$
P\left(k, t_{\ell}, t_{r}, \mu_{r}, \kappa\right)= \begin{cases}1+P\left(k_{L}, t_{\ell}, t_{r}, 1, \kappa-1\right) & \text { if } \mu_{r}=0 \\ 1+P\left(k_{L}, t_{\ell}, t_{r}, \mu_{r}+1, \kappa\right) & \text { if } 0<\mu_{r}<B \\ -\infty & \text { otherwise }\end{cases}
$$

If job $k$ is satisfied in a batch which starts at $t \leq t_{r}-1$, or if $\mu_{r}=0$ and $t \leq t_{r}$, then let $\alpha$ be the number of batches used to satisfy jobs in $L$; this includes the batch satisfying job $k$, so $\alpha>0$. Then $\beta=\kappa-\alpha$ is the budget on the number of batches used to satisfy $R$. Note that for the case where $\mu_{r}=0$, if $R$ is not empty, then any $\alpha$ corresponding to a feasible schedule will be strictly less than $\kappa$, i.e. $\beta>0$.

$$
P\left(k, t_{\ell}, t_{r}, \mu_{r}, \kappa\right)=\left\{\begin{array}{cl}
\max _{0<\alpha \leq \kappa}\left(1+P\left(k_{L}, t_{\ell}, t, 1, \alpha-1\right)\right. & \text { if } 0 \leq \mu_{r}<B \\
\left.+P\left(k_{R}, t, t_{r}, \mu_{r}, \kappa-\alpha\right)\right) & \\
P\left(k, t_{\ell}, t_{r}-1,0, \kappa\right) & \text { otherwise }
\end{array}\right.
$$

Finally, if $\mu_{r}>0$ and $t \in\left(t_{r}-1, t_{r}\right)$, then $P\left(k, t_{\ell}, t_{r}, \mu_{r}, \kappa\right)=-\infty$, since the machine cannot work on multiple batches at the same time. $P\left(k, t_{\ell}, t_{r}, \mu_{r}, \kappa\right)$ is the maximum over these values. Since there are $O(n)$ possible values for $k, O\left(n^{2}\right)$ possible values for both $t_{r}$ and $t_{\ell}$, and $O(n)$ possible values for $\mu_{r}$, and since $\kappa=O(n)$, there are $O\left(n^{7}\right)$ possible values of $P\left(k, t_{\ell}, t_{r}, \mu_{r}, \kappa\right)$ that need to be computed. Computing each one costs $O(n)$, yielding a total running time of $O\left(n^{7} \mathcal{K}\right)$. We note that if the time model is slotted, then there are $O(n)$ possible values for both $t_{r}$ and $t_{\ell}$, and the total running time is instead $O\left(n^{5} \mathcal{K}\right)$. This algorithm can be extended to the case in which each job $J_{k}$ has an associated profit $v_{k}$ and the goal is to schedule a profit-maximizing set of jobs in $\mathcal{K}$ slots.

\section{Disjoint Collection of Windows}

In this section, we discuss the more general problem in which each job's feasible regions $T_{i}$ are arbitrary (as opposed to being a single interval). For any fixed $B \geq 3$, we show that the problem 
is $N P$-hard and discuss its relationship to other classic covering problems. We also develop an efficient algorithm for the two processor case $(B=2)$.

\subsection{Connections to Other Problems}

Capacitated Vertex Cover. There is an interesting relationship between the activation problem of unit-length jobs with multiple feasible windows and the problem of vertex cover with capacity constraints. In the latter problem, we are given a graph $G=(V, E)$ and a capacity function $k(v)$ for each vertex. The goal is to pick a subset $S$ of vertices and to determine an assignment of edges to vertices in $S$, so that each edge is assigned to an incident vertex in $S$, and so that each vertex $v$ has at most $k(v)$ adjacent edges assigned to it. When multiple copies of a vertex may be chosen to be part of the cover, a primal-dual 2-approximation is given in [30. In the hard capacities version of the problem (VCHC), a bounded number of copies of a vertex may be selected. There is a 2-approximation using LP rounding [29, improving the previous bound of 3 given in [14].

In the special case of the activation problem in which each job has exactly two feasible time slots, there is an equivalence with VCHC with uniform capacities $k(v)=B$. Since jobs need not have adjacent feasible time slots, one can view time as a set of slots rather than an ordering of slots. Then, the equivalence follows: the slots are the vertices and the jobs are the edges. For any valid capacity-respecting vertex cover of size $C$, there exists a corresponding activation schedule of $\operatorname{cost} C$ (and vice-versa). One implication of our result is that we can solve VCHC optimally when $k(v)=2$. Furthermore, the 2-approximation for VCHC in [29] applies to the activation problem when each job has two feasible slots. There is a similar relationship between VCHC on hypergraphs and the activation problem where jobs may have more than 2 feasible slots, and for size $g$ hyper edges, an $O(g)$ approximation has recently been developed [52].

Capacitated $K$-center. Another previously studied problem is the $K$-center problem with load capacity constraints [10, 43]. Given an edge weighted graph satisfying the metric property, the goal is to pick $K$ nodes (called centers) and assign each vertex to a chosen center that is "close" to it. We should not assign more than $L$ nodes to any chosen center and want to minimize the value $d_{\max }$ such that each node is assigned to a center within distance $d_{\max }$ of it. Previous work culminated in a 5-approximation for the problem where multiple copies of the same node may be chosen as a center. If only one copy can be chosen then the bound goes up to 6 .

There is the following correspondence between this and the following instance of the activation problem. First, guess the maximum value $d_{\max }$ and create the (unweighted) graph $G^{d_{\text {max }}}$ induced by edges having weight at most $d_{\max }$. Then create a job $J_{i}$ and a timeslot $t_{i}$ for each node $i$ in the original graph $G$, and let job $J_{i}$ be feasible in slot $t_{j}$ if and only if node $i$ is within distance $d_{\max }$ of node (or center) $j$ in $G$, i.e. if edge $(i, j)$ exists in $G^{d_{\max }}$. (So $t_{i}$ should be a feasible slot for $J_{i}$.) Then, finding $K$ centers to which all $n$ vertices can be assigned in $G^{d_{\max }}$ is equivalent to opening $K$ timeslots and feasibly scheduling all $n$ jobs in them so that no slot is assigned more than $B$ jobs, where $B=L$. Since we can solve the activation problem when $B=2$, we can optimally solve the $K$-center problem when $L=2$.

Capacitated Facility Location. Given a set of facilities (with capacities) and clients, the goal is to open a subset of facilities and find a capacity-respecting assignment of clients to open facilities that minimizes the sum of facility opening costs and connection costs. See [13, 46] for approximation algorithms for this problem. Here, we show that when capacities are uniformly 2, the problem can be solved optimally in polynomial time. For clarity's sake, the details of the connection are described at the end of Section 5.3 . 


\subsection{Proof of $N P$-hardness for $\mathrm{B}=3$}

We prove that the activation problem for jobs of arbitrary $T_{i}$ is $N P$-hard when $B=3$ via a reduction from 3 EXACT COVER, which is known to be $N P$-hard [34]. Given a collection $X$ of $n$ elements and a collection of subsets $S_{1}, \ldots, S_{m}$, each containing exactly three elements from $X$. Is there a sub-collection of exactly $\frac{n}{3}$ subsets that exactly cover all of $X$ ? We can view this problem as a bipartite graph where one side has the elements $X$ and the other side has a vertex for each subset $S_{i}$, and edges denote membership. This problem maps to the question of finding a dominating set of size exactly $\frac{n}{3}$ where we are only allowed to select from the side containing subsets. The relationship with the scheduling problem is now obvious: selecting a subset is akin to activating a certain time slot where the set $X$ corresponds to a collection of jobs. The edges specify in which time slots a job can be scheduled. Since the subsets have size exactly 3 , there is an exact cover of size $\frac{n}{3}$ if and only if there is a schedule with exactly $\frac{n}{3}$ active slots.

We observe that there is an $O(\log n)$ approximation for this problem (for any $B$ ) by an easy reduction to a submodular cover problem. This result follows from the classical covering results due to Wolsey [57].

\subsection{Polynomial solution for $\mathrm{B}=2$}

This subsection considers the problem of scheduling jobs in the fewest number of active slots, when there are two processors and each job can be scheduled in a specified subset of time slots. We use the following graph $G$. The vertex set is $J \cup T$, where $J$ is the set of all jobs and $T$ the set of all time slots. The edge set is

$$
\{(j, t): \text { job } j \text { can be scheduled in time slot } t\} \cup\{(t, t): t \in T\} .
$$

A degree-constrained subgraph (DCS) problem is defined on $G$ by the degree constraints $d(j) \leq 1$ for every $j \in J$ and $d(t) \leq 2$ for every $t \in T$. By definition a loop $(t, t)$ contributes two to the degree of $t$.

Any DCS gives a schedule, and any schedule gives a DCS that contains a loop in every nonactive time slot. Also any DCS $D$ that covers $\iota$ jobs and contains $\lambda$ loops has cardinality

$$
|D|=\iota+\lambda
$$

Lemma 1 A maximum cardinality DCS D of G minimizes the number of active slots used by any schedule of $|V(D) \cap J|$ jobs.

Proof: Let $D$ be a maximum cardinality DCS. $D$ covers $\iota=|V(D) \cap J|$ jobs. (4) shows no schedule of $\iota$ jobs contains more loops that $D$. $D$ contains the loop $(t, t)$ precisely when $t$ is not active. Thus $D$ minimizes the number of active slots for schedules of $\iota$ jobs.

To state the time bounds of this section, let $n$ be the number of jobs and $m$ the number of given pairs $(j, t)$ where job $j$ can be scheduled at time $t$. Observe that $G$ has $O(m)$ vertices and $O(m)$ edges, since we can assume every time slot $t$ is on some edge $(j, t)$. In any graph, the majority of edges in a simple non-trivial path are not loops. So in the algorithms for maximum cardinality DCS and maximum matching, an augmenting path has length $O(n)$. A maximum cardinality DCS on $G$ can be found in time $O(\sqrt{n} m)$. The approach is through non-bipartite matching. We describe two ways to handle the loops.

The first approach reduces the problem to maximum cardinality matching in a graph called the $M G$ graph. To do this modify $G$, replacing each time slot vertex $t$ by two vertices $t_{1}, t_{2}$. Replace 
each edge $(j, t)$ by two edges from $j$ to the two replacement vertices for $t$. Finally replace each loop $(t, t)$ by an edge joining the two corresponding replacement vertices.

A DCS $D$ corresponds to a matching $M$ in a natural way: If a time slot $t$ is active in $D$ then $M$ matches corresponding edges of the form $\left(j, t_{i}\right)$. If the loop $(t, t)$ is in $D$ then $M$ matches the replacement edge $\left(t_{1}, t_{2}\right)$. Thus it is easy to see that a maximum cardinality DCS corresponds to a maximum cardinality matching of the same size. The cardinality matching algorithm of Micali and Vazirani [50, 33] gives the desired time bound. (This approach works for the versions of the problem that we will consider on unit jobs; it does not work when the jobs have longer length since a pair of edges $\left(j, t_{1}\right),\left(j, t_{2}\right)$ might get matched.)

A second approach is to use an algorithm for maximum cardinality DCS on general graphs. If such an algorithm does not handle loops directly, modify $G$ as follows by replacing each loop $(t, t)$ by a triangle $\left(t, t_{1}, t_{2}, t\right)$, where each $t_{i}$ is a new vertex with degree constraint $d\left(t_{i}\right)=1$. A DCS in $G$ corresponds to a DCS in the new graph that contains exactly $|T|$ more edges, one from each triangle. The cardinality algorithm of Gabow and Tarjan 32 gives the desired time bound.

Now let $\iota^{*}$ be the greatest number of jobs that can be scheduled. Clearly $\iota^{*}$ can be computed in time $O(\sqrt{n} m)$ by finding a maximum cardinality DCS on the bipartite graph formed by removing all loops from $G$ [20].

Theorem 7 A schedule for the greatest possible number of jobs $\left(\iota^{*}\right)$ that minimizes the number of active slots can be found in time $O(\sqrt{n} m)$.

Proof: The algorithm first finds a DCS $D_{0}$ of $\iota^{*}$ jobs, as described above. It converts $D_{0}$ to the desired DCS by using an algorithm to find a maximum cardinality DCS on $G$, using $D_{0}$ as the initial DCS.

The correctness of this approach follows from the fact that the DCS algorithm works by augmenting paths. This implies that as the initial DCS $D_{0}$ is enlarged to the final DCS, no vertex's degree decreases. Thus the final solution schedules the same jobs as $D_{0}$. Now apply Lemma 1 .

Using $D_{0}$ as the initial DCS does not affect the time bound, since the algorithm begins by finding augmenting paths of length 1, i.e., an arbitrary set of edges satisfying the degree constraints [32, 50].

The proof shows that the choice of $\iota^{*}$ jobs is irrelevant - the minimum number of active slots for a schedule of $\iota^{*}$ jobs can be achieved using any set of $\iota^{*}$ jobs that can be scheduled.

We also note that matching is a natural tool for our power minimization problem: Given a maximum cardinality matching problem, we create a job for every vertex and if two vertices are adjacent, we create a common time slot in which they can be scheduled. A maximum cardinality matching corresponds to a schedule with minimum active time. Thus if $T(m)$ is the optimal possible time for our scheduling problem, a maximum cardinality matching on a graph of $m$ edges can be found in $O(T(m))$ time. For bipartite graphs the number of time slots can be reduced from $m$ to $n$. Thus a maximum cardinality matching on a bipartite graph of $n$ vertices and $m$ edges can be found in $O(T(n, m))$ time, for $T(n, m)$ the optimal time to find a minimum active time schedule for $O(n)$ jobs, $O(n)$ time slots, and $m$ pairs $(j, t)$.

Our algorithm can be extended to other versions of the power minimization problem. For example the following corollary models a situation where power is limited.

Corollary 1 For any given integer $\alpha$, a schedule for the greatest possible number of jobs using at most $\alpha$ active slots can be found in time $O(\sqrt{n} m)$. 
Proof: Start by constructing the DCS $D^{*}$ of Theorem 7 that schedules $\iota^{*}$ jobs and contains say $\lambda^{*}$ loops. Let $\iota^{*}=\tau_{1}+2 \tau_{2}$, where $D^{*}$ schedules $\tau_{i}$ time slots with $i$ jobs, $i=1,2$. Let $\alpha=\tau_{1}+\tau_{2}-\Delta$. We consider the following cases for $\Delta$.

Case $\Delta \leq 0: \quad D^{*}$ has $\leq \alpha$ active slots.

Case $0<\Delta \leq \tau_{1}$ : Choose any $\Delta$ time slots scheduling just one job and unschedule those jobs. This gives a schedule $D$ with $\alpha$ active slots. As a DCS $D$ has $\iota^{*}-\Delta$ jobs and $\lambda^{*}+\Delta$ loops. (4) shows $|D|=\left|D^{*}\right|$. Since fixing the number of active slots fixes $\lambda$, (4) also shows $D$ schedules the greatest possible number of jobs.

Case $\tau_{1}<\Delta$ : In $D^{*}$, unschedule the jobs that are scheduled in the $\tau_{1}$ time slots with one job, or scheduled in $\Delta-\tau_{1}$ other time slots (chosen arbitrarily from the $\tau_{2}$ slots with two jobs). The result is a schedule $D$ with $\alpha$ active slots, each processing two jobs. $D$ obviously schedules the greatest possible number of jobs.

The proof shows that the DCS $D^{*}$ is easily turned into a table (of $\tau_{1}+\tau_{2}$ entries) that gives the desired schedule for every value of $\alpha$.

Now we highlight the connection to capacitated facility location in which capacites are two. Create a weighted graph $M G_{w}$, where jobs correspond to clients and a facility corresponds to a slot, which is a pair of nodes $\left(t_{1}, t_{2}\right)$ in the graph. Add edges $\left(j, t_{1}\right)$ and $\left(j, t_{2}\right)$ with weights equivalent to the cost of connecting client $j$ to facility $t$. Also put an edge between every pair of slot nodes $t_{1}$ and $t_{2}$, with weight $-C(t)$ where $C(t)$ is the cost of opening facility $t$. Then, finding a minimum cost solution to the facility location problem amounts to determining a minimum cost matching in $M G_{w}$ in which each job is scheduled, since the former cost is simply the latter plus an additive term $\sum_{t} C(t)$. The matching can be achieved by generalizing our algorithm to the weighted case in a natural way.

\section{Preemptive Scheduling for Integral Length Jobs}

This section discusses preemptive scheduling (on the integer time points only) for $B=2$. Now each job $j$ has an arbitrary integral length $\ell(j)$, and a set $T_{j}$ of time slots in which one unit of its length can be executed. We wish to assign each job $j$ to exactly $\ell(j)$ of these time slots. again minimizing the number of active time slots. We state several results for this model and sketch their proofs.

Theorem 8 If a schedule executing every job to completion exists, such a schedule minimizing the number of active time slots can be found in time $O(\sqrt{L} m)$ for $L=\sum_{j} \ell(j)$.

Proof Sketch: The algorithm is similar to Theorem [7. We use the graph $G$, modified so job $j$ has degree constraint $d(j) \leq \ell(j)$. Any DCS scheduling every job satisfies (4) for $\iota=L$. The rest of the argument is unchanged. The time bound follows from [32].

Now suppose we cannot schedule all the jobs to completion. Then it is NP-complete to even schedule the greatest possible number of jobs (for any fixed number of processors). We show the proof for $B=1$, the extension for higher $B$ is trivial. The reduction is similar to the NPcompleteness proof in Subsection 3.1 and is as follows: We create a time slot for each element in $X$. We create a collection of $m$ jobs (one corresponding to each set) of length 3 each. Each job can be scheduled in the time slots that correspond to the elements in the corresponding subset. We can schedule $\frac{n}{3}$ jobs if and only if there is a solution to the 3 Exact Cover problem. 
The previous result assumes that $\ell(j)=3$ for all jobs $j$. For $B=2$, the special case where each $\ell(j)$ is 1 or 2 can be modeled by a graph $\widehat{G}$ which is $G$ augmented by a loop $(j, j)$ for every job $j$ of length 2 ; also each such $j$ has degree constraint $d(j)=2$. The following results reduce the problem to maximum weight DCS.

Theorem 9 (a) A schedule for the greatest possible number of jobs, minimizing the number of active slots, can be found in time $O\left(\sqrt{n \alpha(m, m) \log ^{3} m} m\right)$.

(b) For every $\iota_{1}$, a schedule of $\iota_{1}$ unit jobs plus the greatest possible number of length two jobs, minimizing the number of active slots, can be found in the time bound of (a).

(c) The collection of all schedules of (b) (i.e., a schedule for every possible value of $\iota_{1}$ ) can be found in time $O\left(n\left(m+n^{\prime} \log n^{\prime}\right)\right)$, for $n^{\prime}$ the number of jobs and time slots.

Proof Sketch: (a) Assign edge weights to $\widehat{G}$ : An edge $(j, t)$ weighs $m$ if $j$ is unit and $m / 2$ if $j$ has length 2. A loop weighs 1 if it is incident to a time slot and 0 if it is incident to a job. A maximum weight DCS maximizes the number of jobs, and subject to that, maximizes the number of idle time slots. The algorithm of [32] finds a maximum weight DCS in time $O\left(\sqrt{n \alpha(m, n) \log ^{3} n} m\right)$ on a graph of $n$ vertices, $m$ edges, and integral weights polynomial in $n$.

(b) Add a vertex $x$ adjacent to every unit job. Each new edge weighs 0 and $d(x)$, the degree constraint of $x$. is the number of unit jobs decreased by $\iota_{1}$.

(c) In the graph of (b), set $d(x)=0$ and find a maximum weight DCS. Then repeatedly increase $d(x)$ by 1 and update the maximum weight DCS. Each update amounts to finding one augmenting path. The algorithm of [31] finds an augmenting path in time $O(m+n \log n))$ on a graph of $n$ vertices and $m$ edges.

Part (c) is motivated by the fact that unit jobs and length 2 jobs may differ in value. In the same vein, part (b) obviously generalizes to schedules that maximize an arbitrary linear function of the number of unit and length 2 jobs.

\section{Active Time and Arbitrary Preemption}

This section considers the case of scheduling a collection of jobs with arbitrary non-negative lengths $\ell_{j}$ and arbitrary sets $T_{j}$ of feasible intervals, $j \in J$. There are $B$ processors that operate in a collection of unit length time slots $s \in S$. Preemptions are allowed at any time, i.e., job $j$ must be scheduled in $\ell_{j}$ units of time but it can be be started and stopped arbitrarily many times, perhaps switching processors - the only constraint is that it must never execute on more than one processor at any instant of time. We seek a schedule minimizing the active time.

Note that when we allow preemption the multi-slot jobs of Section 5 correspond to arbitrary length jobs. Similarly a special case of this model is preemptively scheduling jobs of arbitrary length with integral release times and deadlines, i.e., the generalization of Section 2 which treats nonpreemptive unit jobs.

\subsection{Linear program formulation}

The problem can be formulated as a linear program. Form a graph of edges $E$ where job $j$ and slot $s$ have an edge $j s$ when $j$ can be scheduled in slot $s$. A variable $x_{j s}$ gives the amount of time job $j$ is scheduled in slot $s$, and a variable $i_{s}$ gives the amount of idle (inactive) time in slot $s$. The problem is equivalent to the following LP: 


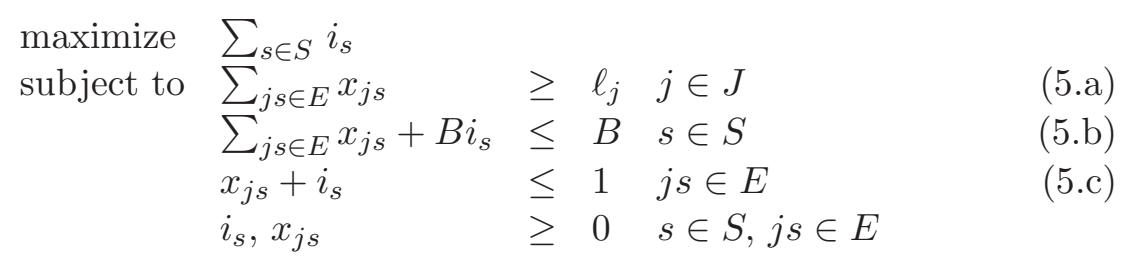

To see the formulation is correct first observe that although (5.a) is an inequality we can assume equality holds, since we need only schedule $\ell_{j}$ units of job $j$.

Secondly, observe that the inequalities (5,b)-(5,c) are necessary and sufficient conditions for scheduling $x_{j s}$ units of job $j, j s \in E$, in $1-i_{s}$ units of time (on $B$ processors). Necessity is clear. For sufficiency, order the jobs arbitrarily; order the processors arbitrarily too. Repeatedly schedule the next $1-i_{s}$ units of jobs on the next processor, until all jobs are scheduled. The last processor may not receive a full $1-i_{s}$ units of work. Also a job may be split across processors. However such a job is scheduled last on one processor and first on the next, so (5.,c) guarantees it is not executed simultaneously on two processors.

We note that the following LP is equivalent to (5). Partition $S$ into disjoint intervals $[a, b)$ where $a$ and $b$ are consecutive integers in the sorted set of interval boundaries over all jobs. Denote these intervals $I_{1}, \ldots, I_{k}$ and let $E^{\prime}$ be the set of edges $i j$ where job $j$ is feasible in $I_{i}$.

$$
\begin{array}{llll}
\operatorname{maximize} & \sum_{i=1}^{k} y_{i} & & \\
\text { subject to } & \sum_{i j \in E^{\prime}} x_{i j} & \geq \ell_{j} & j \in J \\
& \sum_{i j \in E^{\prime}} x_{i j}+B \cdot y_{i} & \leq B \cdot\left|I_{i}\right| & i=1 \ldots k \\
& x_{i j}+y_{i} & \leq\left|I_{i}\right| &
\end{array}
$$

The variable $y_{i}$ denotes the amount of idle time in interval $I_{i}$. The variable $x_{i j}$ denotes the amount of time devoted to job $j$ in interval $I_{i}$. For jobs of arbitrary length, the size of this LP is still polynomial in the number of jobs and the total number of intervals in the sets $T_{j}$.

\subsection{The case $B=2$ and 2-matchings}

It is convenient to use a variant of (5) based on the matching graph $M G$ of Section 5 . Each edge of $M G$ has a linear programming variable. Specifically each edge $j s \in E(G)$ gives rise to two variables $x_{e}, e=j s_{i}, i \in\{1,2\}$, that give the amount of time job $j$ is scheduled on processor $i$ in time slot $s$. Also each time slot $s \in S$, has a variable $x_{e}, e=s_{1} s_{2}$ that gives the amount of inactive time in slot $s$. The problem is equivalent to the following LP:

$$
\begin{array}{lll}
\operatorname{maximize} & \sum\left\{x_{e}: e \in E(M G)\right\} \\
\text { subject to } & \sum\left\{x_{e}: e \text { incident to } j\right\} & =\ell_{j} \quad j \in J \\
& \sum\left\{x_{e}: e \text { incident to } s_{i}\right\} & \leq 1 \quad s \in S, i \in\{1,2\} \\
& \sum\left\{x_{e}: e \in\left\{j s_{1}, j s_{2}, s_{1} s_{2}\right\}\right\} \leq 1 \quad j s \in E(G)
\end{array}
$$

To see this formulation is correct note that any schedule supplies feasible $x_{e}$ values. Conversely any feasible $x_{e}$ 's give a feasible solution to LP (5) (specifically set $x_{j s}=x_{j s_{1}}+x_{j s_{2}}$ and $i_{s}=x_{s_{1} s_{2}}$ ) 


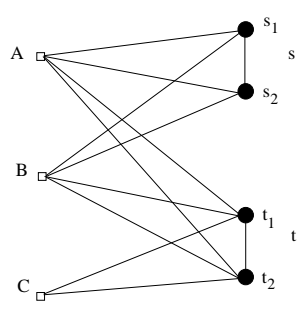

(a)

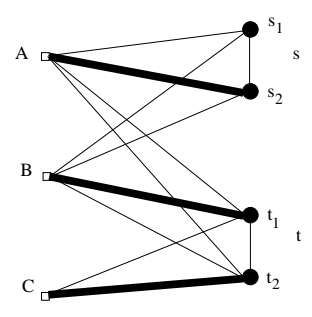

(b)

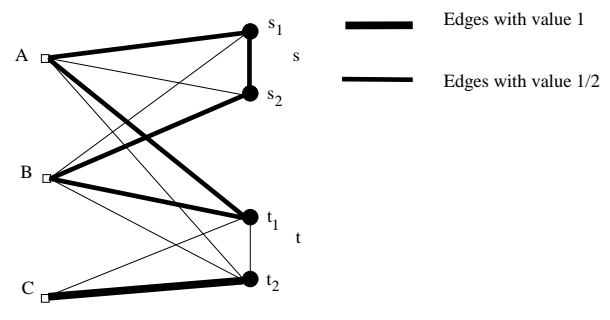

(c)

Figure 2: (a) We illustrate the graph $M G$ with two time slots and three jobs with $B=2$. Jobs A and $\mathrm{B}$ can be scheduled in time slots s and $\mathrm{t}$, while job $\mathrm{C}$ can only be scheduled in time slot t. (b) We show a solution corresponding to an integral matching and (c) a solution corresponding to a triangle free 2-matching with $\frac{1}{2}$ unit of idle time in the first slot.

and so corresponds to a schedule. Finally the objective of (7) equals the total of all job lengths plus the total inactive time, so like (5) it maximizes the total inactive time.

Now consider an arbitrary graph $G=(V, E)$ and the polyhedron defined by the following system of linear inequalities:

$$
\begin{array}{lll}
\sum\left\{x_{e}: e \text { incident to } v\right\} & \leq 1 \quad v \in V \\
\sum_{x_{e}}\left\{x_{e}: e \text { an edge of } T\right\} & \leq 1 \quad T \text { a triangle of } G \\
& \geq 0 \quad \text { (8, }, \mathrm{b})
\end{array}
$$

Call $\sum\left\{x_{e}: e \in E\right\}$ the size of a solution to (8). Say vertex $v$ is covered if equality holds in (8, a).

Define a 2-matching $M$ to be an assignment of weight 0,1 or $1 / 2$ to each edge to each edge of $G$ so that each vertex is incident to edges of total weight $\leq 13 M$ is basic if its edges of weight $1 / 2$ form a collection of (vertex-disjoint) odd cycles. The basic 2-matchings are precisely the vertices of the polyhedron determined by the constraints (8) with (8,b) omitted. A basic 2-matching is triangle-free if no triangle has positive weight on all three of its edges. Cornuéjols and Pulleyblank [16] showed the triangle-free 2-matchings are precisely the vertices of the polyhedron determined by constraints (8).

When all job lengths are one, the inequalities of (17) are system (8) for graph $M G$, with the further requirement that every job vertex is covered. So it is easy to see that the result of Cornuéjols and Pulleyblank implies our scheduling problem is solved by any triangle-free 2-matching on $M G$ that has maximum size subject to the constraint that it covers every job vertex. Also, interestingly, there is always solution where each job is scheduled either completely in one time slot or is split into two pieces of size $1 / 2$ (see Fig. 2).

Cornuejols and Pulleyblank give two augmenting path algorithms for triangle-free 2-matching: they find such a matching that is perfect (i.e., every vertex is fully covered) in time $O(n m)$ [17], and such a matching that has minimum cost in time $O\left(n^{2} m\right)$ [16]. (The latter bound clearly applies to our scheduling problem, since it can model the constraint that every job vertex is covered.) Babenko et. al. [5] showed that a maximum cardinality triangle-free 2-matching can be found in time $O(\sqrt{n} m)$. This is done by reducing the problem to ordinary matching, with the help of the Edmonds-Gallai decomposition.

\footnotetext{
${ }^{3}$ This definition of a 2 -matching scales the usual definition by a factor $1 / 2$, i.e., the weights are usually 0,1 or 2 .
} 


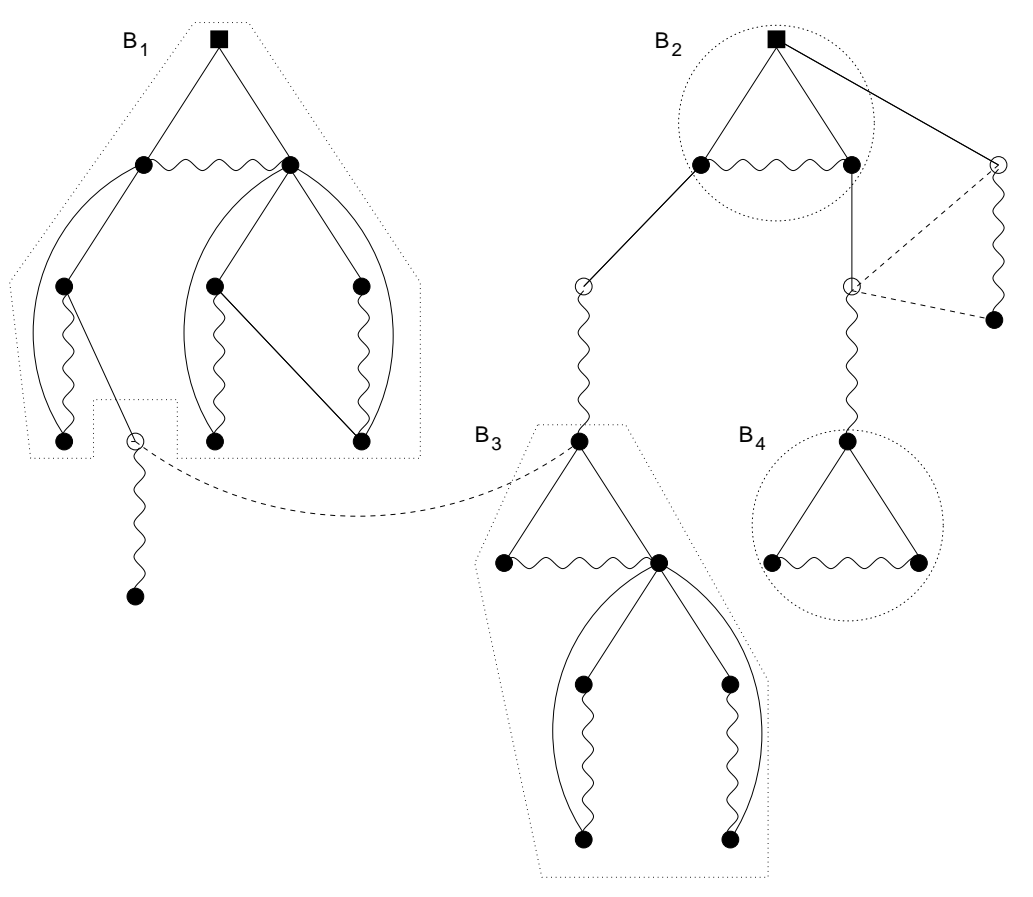

Figure 3: Matching with four blossoms.

Here we give two easy extensions of [5]: a simple application of the Mendelsohn-Dulmage Theorem [45] shows that a maximum cardinality triangle-free 2-matching can be found in the same asymptotic time as a maximum cardinality matching (e.g., the algebraic algorithm of Mucha and Sankowski [51] can be used). This result extends to maximum cardinality triangle-free 2-matchings that are constrained to cover a given set of vertices (this is the variant needed for our scheduling problem). The development is based on standard data structures for blossoms. For instance, it is based on a simple definition of an augmenting cycle (analog of an augmenting path), leading to an augmenting blossom (which models the triangle cluster of Cornuejols and Pulleyblank); we show a maximum cardinality matching with the greatest number of augmenting blossoms gives a maximum cardinality cardinality triangle-free 2-matching (Lemma 4(i)) 4

Our proof shows that the scheduling problem is solved correctly, independently of [16, 17, [5]. We also extend the ideas to get similar results for arbitrary job lengths.

\subsection{Triangle-free 2-matchings}

This section gives an efficient algorithm to find a maximum cardinality triangle-free 2-matching.

The approach to 2-matchings is through ordinary matchings. Fig. 3 illustrates the discussion. Wavy edges are matched. Square vertices are unmatched.

All paths and cycles that we consider are simple. A path is alternating if its edges are alternately matched and unmatched; a cycle is alternating if one vertex is incident to two unmatched edges and otherwise the edges are alternately matched and unmatched.

Consider a matching $M$ on an arbitrary graph $G$. An augmenting cycle $A$ is an alternating odd cycle of length $>3$ that contains an unmatched vertex (i.e., a vertex $\notin V(M)$ ). Changing the weight of every edge of $A$ to $1 / 2$ gives a 2 -matching of size $|M|+1 / 2$. We will show that a maximum

\footnotetext{
${ }^{4}$ Our algorithm was developed independently of the authoritative algorithm of [5].
} 
size triangle-free 2-matching, and a solution to our scheduling problem, can be constructed using augmenting cycles.

We assume some familiarity with the blossom algorithm for maximum cardinality matching [45] but we review most notions.

A blossom is a recursively defined subgraph $B$. The vertices of $B$ are partitioned into an odd number of subgraphs $B_{i}$. Each $B_{i}$ is either a single vertex or a blossom. If we contract each $B_{i}$ to a vertex, the remaining edges of $B$ form an alternating odd cycle spanning every $B_{i}$. Fig. 3 has four maximal blossoms $B_{1}-B_{4}$.

Let $B_{0}$ be the unique $B_{i}$ that is incident to two unmatched edges of $B$. The base (vertex) $b$ of $B$ is $B_{0}$ if $B_{0}$ is a vertex, else it is the base of $B_{0}$. An easy induction shows that every vertex of $B-b$ is on a matched edge of $B$. If $b$ is on a matched edge, that edge is incident to $V(B) . B$ is a matched (unmatched) blossom if $b$ is on some (no) matched edge.

Two well-known properties of blossoms can be shown by simple induction:

P1: Let $B$ be a blossom with base $b$. For any $v \in V(B), B$ contains an even length alternating path from $v$ to $b$.

P2: Let $B$ be an unmatched blossom. The matching on $E(B)$ can be modified to make any given vertex of $B$ the base.

A triangle cluster [16, 17] is is a connected subgraph whose biconnected components are all triangles. Note that a single vertex is a triangle cluster. Also, a subgraph is a triangle cluster exactly when it is connected, its edges can be partitioned into triangles, and any vertex shared by two or more triangles is a cutpoint. A blossom whose vertices induce a subgraph that is a triangle cluster is a t-blossom ( $B_{2}-B_{4}$ in Fig. [3); in the opposite case it is a non-t-blossom. A non-t-blossom is augmenting if it is unmatched $\left(B_{1}\right.$ in Fig 3$)$.

Lemma 2 The matching on an augmenting blossom $B$ can be modified so $V(B)$ is spanned by a set of matched edges and an augmenting cycle.

Proof: Consider an arbitrary blossom $A$. Its recursive definition gives various subblossoms $B$, each composed of subgraphs $B_{i}, i=0, \ldots, k, k$ even, along with edges $u_{i} v_{i+1}, i=0, \ldots, k$ joining $B_{i}$ to $B_{i+1}$. (Take $k+1$ to be 0 .) Consider one of these subblossoms $B$.

Make $u_{0}$ the base of $A$ (by $\mathrm{P} 2$ ). Let $P$ be the even length alternating path in $B$ from $v_{1}$ to the base $u_{0}$ (by P1). Adding edge $u_{0} v_{1}$ to $P$ completes an alternating cycle $C$. $A$ is spanned by $C$ and the matched edges of $A-C$. In the first two cases below $B$ can be chosen so $|C| \geq 5$, i.e., $C$ is the desired augmenting cycle.

Case 1: Some subblossom $B$ has $k>2$. Use $B$ in the above procedure. Note that $C$ goes through every $B_{i}$. (It starts in $B_{1}$, so to reach $u_{0} \in B_{0}$ it must go through $B_{2}, \ldots, B_{k}, B_{0}$ in that order.) Thus $C$ goes through $\geq 5$ subblossoms and $|C| \geq 5$.

Case 2: Some subblossom $B$ has some $u_{i} \neq v_{i}$. $C$ traverses a path in $B_{i}$ from $v_{i}$ to $u_{i}$. So it has $\geq 2$ vertices in $B_{i}$ and $\geq 1$ vertex in every other subblossom, i.e., $\geq 4$ vertices. Since $C$ has odd length, $|C| \geq 5$.

Case 3: Every subblossom $B$ has $k=2$ as well as $u_{i}=v_{i}$ for every $i$. Every subblossom consists of three edges forming a triangle. So the edges of $A$ form a triangle cluster.

Suppose $A$ is an augmenting blossom. So $A$ does not induce a triangle cluster, i.e., some edge $u v \in E(G)-E(A)$ joins two vertices of $A$. Make $u$ the base of $A$. Let $P$ be the even length 


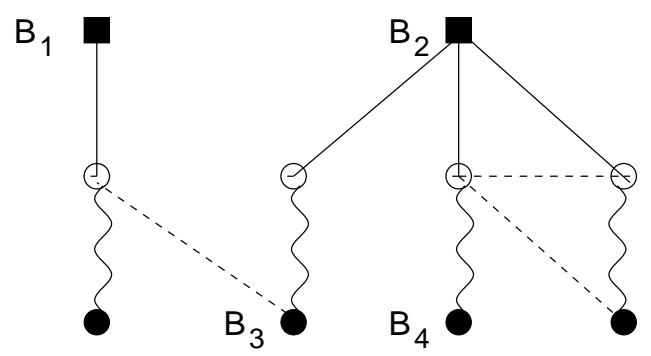

Figure 4: Hungarian subgraph for the graph in Fig. 3 .

alternating path in $A$ from $v$ to $u$ (by P1). Adding edge $u v$ to $P$ completes an alternating cycle $C$. $A$ is spanned by $C$ and the matched edges of $A-C$. $C$ is not a triangle since it contains edge $u v$ not in the triangle cluster. Thus $|C| \geq 5$.

We use some more concepts from ordinary matching [45]. An alternating tree has an unmatched root, every path from the root is alternating, and every leaf has even distance from the root. A vertex at even (odd) distance from the root is outer (inner).

Fig. 4 illustrates the following definition (for the matching of Fig. 3): Let $M$ be a maximum cardinality matching on $G$. A Hungarian subgraph $H$ for $M$ has nodes that are either vertices of $G$ or contractions of blossoms in $G$. No vertex of $G$ occurs more than once as a node of $H$ or as a member of a blossom. Every unmatched vertex of $G$ is either a node of $H$ or the base of an unmatched blossom of $H$. $H$ is spanned by a forest of alternating trees. Each blossom $B$ of $H$ is outer in this forest. Call a vertex of $G$ outer if it is an outer node of $H$ or in some blossom of $H$, and inner if it is an inner node. We sometimes write $V_{H}$ to denote the set of all inner and outer vertices of $G$. The key property is:

(*) Any edge $v w$ of $G$ with $v$ an outer vertex has $w$ inner or $v$ and $w$ in the same blossom.

In Fig. 4 (as well Fig. 3) inner vertices are hollow, outer are filled. The three dashed edges are non-tree edges.

We use a slight extension of this concept: Take $G, M, H$ as before. Let $\mathcal{B}$ be the set of augmenting blossoms in $H$. (We shall see below that $\mathcal{B}$ does not depend on choice of Hungarian subgraph $H$.) Let $T(M)$ be the triangle-free 2-matching of size $|M|+|\mathcal{B}| / 2$ formed by using Lemma 2 on each blossom of $\mathcal{B}$ and enlarging $M$ in the obvious way. A reduced Hungarian subgraph $H$ for $T(M)$ is a Hungarian subgraph on $G-\bigcup\{V(B): B \in \mathcal{B}\}$ for $M$ restricted to this graph. We reiterate that a vertex of an augmenting blossom does not belong to $V_{H}$. The reduced Hungarian subgraph for the matching of Fig. 3 is Fig. 4 with $B_{1}$ deleted, i.e., the leftmost dashed edge becomes a tree edge.

Lemma 3 A reduced Hungarian subgraph for $T(M)$ satisfies $(*)$.

Proof: For $(*)$ to fail an edge must join an outer vertex $v$ to an augmenting blossom $B$. This edge completes an augmenting path for $M$, from the unmatched vertex of $B$ to the root of $v$ 's alternating tree. But this contradicts the maximum cardinality of $M$.

This section uses the notion from matching theory of "set cover" (as in odd set covers, reviewed below). A set cover $\mathcal{C}$ for an arbitrary graph $G$ is a subpartition of the vertices such that every edge of $G$ either has both ends in the same set of $\mathcal{C}$ or at least one end in a singleton set of $\mathcal{C}$. We use several types of these covers, each with its own definition of "capacity" of a set. The intent is that 
the capacity $\mathcal{C}$ (i.e., the total capacity of all its sets) should upper bound the size of a matching of some type.

In a triangulated set cover $\mathcal{C}$, the capacity of a set $S \in \mathcal{C}$ is 1 for a singleton, $\lfloor|S| / 2\rfloor$ if $E(S)$ is a triangle cluster and $|S| / 2$ otherwise. The total weight of any solution to LP (8) is at most the capacity of any such cover $\mathcal{C}$. In proof a singleton is incident to edges of total weight $\leq 1$ (its capacity). Let $S \subseteq V$ be a non-singleton. The total weight of all edges with both ends in $S$ is $\leq|S| / 2$. Finally suppose $S$ induces a triangle cluster of $t$ triangles. $S$ has $2 t+1$ vertices, and the edges with both ends in $S$ weigh at most $t=\lfloor|S| / 2\rfloor$.

The 2-matchings we wish to construct are characterized as follows:

Lemma 4 (i) If $M$ is a maximum cardinality matching with the greatest possible number of augmenting blossoms in its Hungarian subgraph, then $T(M)$ is a maximum size triangle-free 2matching. In fact it is a maximum size solution to (8).

(ii) Let $J$ be a set of vertices that can be covered by a triangle-free 2-matching. Let $M$ be a maximum cardinality matching with the greatest possible number of augmenting blossoms in its Hungarian subgraph, subject to the constraint that every vertex of $J$ is matched or in an augmenting blossom. If such an $M$ exists, $T(M)$ is a maximum size triangle-free 2-matching that covers $J$. In fact it is a maximum size solution to (8) when every vertex of $J$ is constrained to have equality in (8. a).

Proof: $(i)$ Let $H$ be a reduced Hungarian subgraph for $T(M)$. Let $B$ be any blossom in $H$. A maximum cardinality matching with $B$ unmatched can be formed (by interchanging the matched and unmatched edges in the path from $B$ to the root of its alternating tree). This makes $B$ an augmenting blossom if it is a non-t-blossom. So the choice of $M$ makes $B$ a t-blossom.

Consider the family of sets

$$
\mathcal{C}=\left\{V-V_{H},\{v\}, V(B): v \text { an inner vertex, } B \text { a blossom of } H\right\} .
$$

$\mathcal{C}$ is a set cover (property $(*)$ shows edges with an outer end are handled correctly). The size of $T(M)$ equals the capacity of $\mathcal{C}$ considered a triangulated set cover (since no edge of positive weight leaves $V-V_{H}$, any inner vertex is on a weight 1 edge leading to an outer vertex, and the number of weight 1 edges with both ends in a blossom $B$ of $H$ is $\lfloor|V(B)| / 2\rfloor$, which equals the capacity of $V(B)$ as a triangle cluster). Part $(i)$ follows.

(ii) $T(M)$ covers $J$ since it covers every vertex in an augmenting blossom of $M$. The rest of the argument is identical to part $(i)$.

We turn to the algorithm to find a maximum cardinality triangle-free 2-matching. First recall two more ideas from matching theory. An odd set cover $\mathcal{C}$ for an arbitrary graph $G$ is set cover (as defined above) where the capacity of a set $S \in \mathcal{C}$ is 1 for a singleton, else $\lfloor|S| / 2\rfloor$ [45, 49].

It is easy to see that the cardinality of any matching is at most the capacity of any odd set cover. Furthermore equality can always be achieved: Take any Hungarian subgraph $H$ for any maximum cardinality matching $M$. An argument similar to the previous one shows that $|M|$ equals the capacity of the odd set cover

$$
\left\{V-V_{H},\{v\}, V(B): v \text { an inner vertex, } B \text { a blossom }\right\} .
$$

Next we recall the Edmonds-Gallai decomposition, which gives the structure of any maximum cardinality matching. (Parenthetic remarks will sketch proofs that this decomposition is correct. Let $\mathcal{C}$ be an odd set cover whose capacity is the size of a maximum cardinality matching.) Call 
a matching perfect on $S \subseteq V$ if every vertex of $S$ is matched with another vertex of $S$, and nearperfect if this holds for all but one vertex of $S$. (So $|S|$ is even in the first case and odd in the second.)

Start with a Hungarian subgraph $H$ for some maximum cardinality matching. (For our proofs, let $\mathcal{C}$ be the odd set cover constructed from $H$ as described above.) We will describe the structure of an arbitrary maximum cardinality matching $M$.

(a) $M$ contains a perfect matching of the vertices not in $V_{H}$. (This follows since $V-V_{H} \in \mathcal{C}$.) (b) $M$ contains a near-perfect matching of any blossom $B$ of $H$. (This follows since $B \in \mathcal{C}$.)

(c) $M$ contains a maximum cardinality matching of the nodes of $H$. (This follows from the maximum cardinality of $M$ and (a)-(b).) In fact this matching is a maximum cardinality matching of the bipartite graph $B G$ whose edge sets are the inner nodes, and the outer nodes of $H$ and whose edges are the edges of $H$ that join inner node to outer. (This follows since $\mathcal{C}$ shows $M$ does not contain any edge joining two inner vertices.) In Fig $4 B G$ does not contain the horizontal dashed edge.

We remark that the matching of $(\mathrm{c})$ covers every inner vertex (again by $\mathcal{C}$ ). Note that each edge of $B G$ can be identified with one or more edges of $G$. Once this edge is chosen the matching of (c) determines the unmatched vertices in the near-perfect matchings of (b). So starting from any maximum cardinality matching $B M$ of $B G$, we can construct a corresponding matching of $G$ satisfying (a)-(c), i.e., a maximum cardinality matching of $G$.

The last part of Edmonds-Gallai states that the sets $V(B)$ for blossoms $B$ of $H$ are an invariant of $G$. (In proof, property $(*)$ implies the outer vertices of $H$ are precisely those vertices that can be reached from an unmatched vertex (of $M$ ) by an even length alternating path. Thus the outer vertices constitute the set $U$ of vertices that are unmatched in some maximum cardinality matching. The sets $V(B)$ are the non-singleton connected components of $U$.)

In the rest of this section, $O$ denotes the set of outer nodes in the above bipartite graph $B G$, and $N \subseteq O$ denotes its set of non-t-blossoms.

Lemma 5 (i) Let $B M$ be a maximum cardinality matching of $B G$ that matches the greatest possible number of nodes of $O-N$. Then $T(M)$ is a maximum size triangle-free 2-matching if $M$ is a maximum cardinality matching of $G$ corresponding to $B M$ (as described above).

(ii) Let $J$ be a set of vertices that can be covered by a triangle-free 2-matching. Let BM be a maximum cardinality matching of $B G$ that matches every node of $O-N$ that is a subset of $J$, and as many other nodes of $O-N$ as possible. Then $T(M)$ is a maximum size triangle-free 2-matching that covers $J$ if $M$ is a maximum cardinality matching of $G$ corresponding to $B M$ such that no base vertex of an unmatched blossom of $O-N$ belongs to $J$.

Proof: $(i)$ Let $B M^{*}$ be a matching on $B G$ that corresponds to a maximum cardinality matching of $G$ having the greatest possible number of augmenting blossoms. The invariance of blossoms implies that the augmenting blossoms of $B M^{*}$ are precisely the blossoms of $N$ that are unmatched in $B M^{*}$. By Lemma 4 we need only show that $B M$ has as many augmenting blossoms as $B M^{*}$.

The size of a matching on $B G$ is the number of matched outer nodes, so $|O \cap V(B M)|=$ $\left|O \cap V\left(B M^{*}\right)\right|$. By definition $|(O-N) \cap V(B M)| \geq\left|(O-N) \cap V\left(B M^{*}\right)\right|$. So $|N \cap V(B M)| \leq$ $\left|N \cap V\left(B M^{*}\right)\right|$. This implies the desired inequality $|N-V(B M)| \geq\left|N-V\left(B M^{*}\right)\right|$.

(ii) Suppose

(a) the matching $B M$ of part (ii) exists, and

(b) matching $M$ of part (ii) exists and covers every vertex of $J$. 
Then part (ii) follows using the argument for part $(i)$.

To establish (a)-(b), let $J_{0}$ denote the set of nodes of $O-N$ that are subsets of $J$.

Claim $B G$ has a matching $B M_{0}$ that covers every node of $J_{0}$.

The claim implies (a). To show it implies (b), Edmonds-Gallai shows any unmatched node of $B M$ is outer. The claim shows any blossom of $O-N$ that is unmatched in $B M$ contains a vertex $v \notin J$. $v$ can be made the unique unmatched vertex of $B(\mathrm{P} 2)$. So $M$ covers $J-N$, as does $T(M)$. $T(M)$ covers every vertex in a blossom of $N$. (b) follows.

To prove the claim let $M^{2}$ be a triangle-free 2-matching that covers $J$, viewed as a collection of matched edges and odd cycles. We will construct a set of edges $S \subseteq M^{2}$ such that each outer node covered by $M^{2}$ has degree $\geq 1$ in $S$, and each inner node has degree $\leq 1$. Clearly $S$ contains the desired matching $B M_{0}\left(B M_{0}\right.$ is a subset of $B G$ by property $\left.(*)\right)$.

We construct $S$ by traversing the connected components of $M^{2}$, maintaining the invariant that any outer node covered by a traversed edge of $M^{2}$ has degree $\geq 1$ in $S$ and any inner node has degree $\leq 1$ in $S$. We traverse a component $C$ of $M^{2}$ as follows.

Suppose $C$ is a matched edge. Add it to $S$. Clearly this preserves the invariant.

Suppose $C$ is an odd cycle. The edges of $C \cap B G$ form a number of connected components. Traverse each such component, adding alternate edges to $S$. An outer node $x$ in such a component is on $\geq 1$ edge added to $S$, since $C$ leaves $x$ each time it enters it, and property $(*)$. An inner node $x$ maintains degree $\leq 1$ in $S$, since $x$ is on just 2 edges of $C$ (each of which may or may not be in $B G)$. The claim follows.

In summary the algorithm to find a maximum cardinality triangle-free 2-matching works as follows. Find a maximum cardinality matching $M_{0}$ of $G$ and its Hungarian subgraph $H$. Use $H$ to construct $B G$. Find a maximum cardinality matching $M_{1}$ of $B G-N$. Find a maximum cardinality matching $M_{2}$ of $B G$ that covers all nodes covered by $M_{1}$. Extend $M_{2}$ to a maximum cardinality matching $M_{3}$ of $G$, using (a)-(c). Convert $M_{3}$ to the 2-matching $T\left(M_{3}\right)$ by reweighting the augmenting blossoms. Return $T\left(M_{3}\right)$.

If the 2-matching is required to cover a set of vertices $J$ two simple changes are needed: Take $M_{1}$ as a maximum cardinality matching of $B G-N$ subject to the constraint that it matches every node of $O-N$ that is a subset of $J$. Take $M_{3}$ so it leaves a vertex not in $J$ unmatched in each unmatched blossom of $O-N$.

Theorem 10 A maximum cardinality triangle-free 2-matching can be found in time $O(\sqrt{n} m)$. The same holds if the 2-matching is constrained to cover a set of vertices $J$, assuming $J$ can be covered by some triangle-free 2-matching.

Proof: The maximum cardinality matchings are found in time $O(\sqrt{n} m)$ [50, 33]. A Hungarian subgraph for a given maximum cardinality matching can be found in time $O(m)$ [27, 33]. Hence the total time for both of our algorithms is $O(\sqrt{n} m)$.

Corollary 2 A maximum cardinality triangle-free 2-matching can be found in the same asymptotic time as a maximum cardinality matching. The same holds if the 2-matching is constrained to cover a set of vertices $J$.

Proof: The argument for a 2-matching constrained to cover $J$ is essentially the same as the unconstrained case, so we discuss only the latter. We show that excluding the time for maximum cardinality matching, our algorithm uses time $O(m)$. 
As just noted $H$ is constructed in $O(m)$ time in [27, 33]. This construction also supplies the recursive decomposition of each blossom $B$ into subblossoms $B_{i}$, so it is easy to find the near-perfect matchings of (b) in Edmonds-Gallai (for $M_{3}$ ). It is also easy to classify each blossom of $H$ as a triangle cluster or a non-t-blossom.

It only remains to describe how to find the maximum cardinality matching $M_{2}$ of $B G$ that covers all nodes covered by $M_{1}$. The following well-known procedure works for an arbitrary matching $M_{1}$ on an arbitrary graph $G$.

Start by finding any maximum cardinality matching $M_{2}$ of $G$. The edges of $M_{1} \cup M_{2}$ form a number of connected components, each of which is a path or cycle with edges alternating between the two matchings. A node $x \in V\left(M_{1}\right)-V\left(M_{2}\right)$ is the end of a component that is an alternating path $P$. $P$ has even length and its other end $y$ is in $V\left(M_{2}\right)-V\left(M_{1}\right)$ (since $M_{2}$ has maximum cardinality). So replacing the edges of $P \cap M_{2}$ by $P \cap M_{1}$ makes $x$ covered and keeps $M_{2}$ maximum cardinality. ( $y$ becomes uncovered but this is not a problem.) Doing this for all such $x$ makes $M_{2}$ the desired maximum cardinality matching. Clearly the entire construction uses time $O(n)$.

It is well-known that the problems of maximum size 2-matching and maximum cardinality bipartite matching have the same asymptotic time bound. It is also clear that finding a maximum size triangle-free 2-matching is at least as hard as maximum cardinality bipartite matching.

\subsection{Applications to preemptive scheduling for $B=2$}

The algorithm of Theorem 10, along with the LP (7), solves the preemptive scheduling problem for unit jobs. We begin by extending the solution to arbitrary job lengths $\ell_{j}$.

We reduce the general problem to the unit length case, using a graph $U G$ with unit jobs defined as follows. As before each time slot $s$ is represented by vertices $s_{1}, s_{2}$ and edge $s_{1} s_{2}$. A job $j$ of length $\ell_{j}$, which may be scheduled in a set of slots $S_{j}$, is represented by vertices $u_{j s}, s \in S_{j}$, each with two edges $u_{j s} s_{i}, i=1,2$. In addition job $j$ has vertices $\bar{u}_{j i}, i=1, \ldots,\left|S_{j}\right|-\ell_{j}$. with a complete bipartite graph joining its two types of vertices $u_{j s}$ and $\bar{u}_{j i}$.

The triangles of $U G$ have the form $u_{j s}, s_{1}, s_{2}$ and are similar to those of $M G$. Define the sets of vertices $J_{U}=\left\{u_{j s}: j \in J, s \in S_{j}\right\}, \bar{J}_{U}=\left\{\bar{u}_{j i}: j \in J, i \leq\left|S_{j}\right|-\ell_{j}\right\}$. It is easy to see that a solution to LP (8) on graph $U G$ that covers every vertex of $J_{U} \cup \bar{J}_{U}$ gives a feasible solution to LP (77) and vice versa. So as before [17] (or Lemma [4(ii)) shows the solution to our problem is given by a triangle-free 2-matching on $U G$ that covers $J_{U} \cup \bar{J}_{U}$ and has the greatest cardinality possible.

Theorem 11 Let $J$ be a set of unit jobs that can be scheduled on $B=2$ processors. A preemptive schedule for $J$ minimizing the active time can be found in $O(\sqrt{n} m)$ time. The result extends to jobs of arbitrary integral lengths $\ell_{j}$, where the time is $O(\sqrt{L} m)$ for $L$ the sum of all job lengths.

Proof: The algorithm for triangle-free 2-matching (Theorem 10) can be implemented in time $O(\sqrt{L} m)$ on graph $U G$. To do this we use the vertex substitution technique of [26] which works on graphs of $O(m)$ edges rather than $U G$ itself.

A standard construction from network flow shows that regarding feasibility, allowing arbitrary preemption does not help in this sense: For any number of processors, a set of jobs that can be feasibly scheduled with arbitrary preemption can also be scheduled limiting preemption to integral time points, assuming the jobs are either all unit length, or they have arbitrary integral lengths $\ell_{j}$ and time is slotted. (In detail use a flow graph where job $j$ is a source of capacity $\ell_{j}$, slot $t$ is a sink of capacity $p$, and edge $j t$ exists when $j$ can be scheduled in slot $t$. The Integrality Theorem shows we can assume each $j$ gets scheduled in exactly $\ell_{j}$ time slots.) 
However, even in those situations, arbitrary preemption can reduce active time. For example when $B=2$, three unit jobs that may be scheduled in slots 1 or 2 require 2 units of active time using preemption at integer times and only $3 / 2$ units with arbitrary preemption. We now show this example gives the greatest disparity between the two preemption models for $B=2$.

Recall that we construct an optimal preemptive schedule $\mathcal{P}$ by finding a a maximum size trianglefree 2-matching as specified in Lemma 4 (ii) (on $M G$ for unit jobs, and $U G$ for arbitrary length jobs) and converting it to $\mathcal{P}$ in the obvious way via LP (17). Part (iii) of the lemma below gives the main property for establishing the desired bound. The following lemma is proved by examining the structure of blossoms in our special graphs, e.g., the triangles all have the form $j s_{1} s_{2}$ for $j$ a job vertex and $s_{1} s_{2}$ the edge representing a time slot; also vertices $s_{1}$ and $s_{2}$ are isomorphic.

Lemma 6 (i) A blossom in $M G(U G)$ is a triangle cluster iff it contains exactly one vertex of $J$ $\left(J_{U}\right)$, respectively.

(ii) Let $H$ be a Hungarian subgraph in $M G$ or $U G$. Any slot s either has both its vertices inner, or both its vertices outer (and in the same blossom) or neither vertex in $V_{H}$.

(iii) Let the optimal preemptive schedule $\mathcal{P}$ be constructed from $T(M)$, and let $B$ be an augmenting blossom of $M$. The time slots with both vertices in $B$ have $\geq 3 / 2$ units of active time in $\mathcal{P}$.

Proof: $(i)$ A triangle $T$ in $M G$ or $U G$ must contain a slot edge $s_{1} s_{2}$. So the other two edges must be $j s_{1}, j s_{2}$ for vertex $j \in J \cup J_{U}$. A triangle $T^{\prime}$ that shares a vertex but no edge with $T$ must contain vertex $j$. So the triangle clusters are sets of edges $j s_{1}, j s_{2}, s_{1} s_{2}$ where $j$ is a fixed vertex of $J \cup J_{U}$. (In $U G$ a cluster has only one triangle.)

Clearly this implies any t-blossom has exactly one vertex of $J \cup J_{U}$.

Conversely let $B$ be a non-t-blossom. Make $B$ an augmenting blossom by unmatching any edge incident to its base. Then Lemma 2 shows $B$ has an alternating cycle $C$ of $\geq 5$ edges. $C$ must contain an edge of the form $s_{1} s_{2}$. The two other edges of $C$ incident to $s_{1}$ and $s_{2}$ must go to vertices of $J \cup J_{U}$ that are distinct.

(ii) First observe that $s_{1} \in V_{H}$ implies $s_{2} \in V_{H}$ : If $s_{1}$ is outer this follows from edge $s_{1} s_{2}$. If $s_{1}$ is inner then its parent contains an outer vertex $j \in J \cup J_{U}$ adjacent to $s_{1} \cdot j$ is also adjacent to $s_{2}$ so $s_{2} \in V_{H}$.

We complete the proof by showing that if $s_{1}$ is outer then so is $s_{2}$. If, on the contrary, $s_{2}$ is inner then it is adjacent to an outer vertex in its parent and an outer vertex in its child. These vertices are obviously in different blossoms. But $s_{1}$ is adjacent to both of them, so both are in the blossom containing $s_{1}$, contradiction.

(iii) First consider the case of unit jobs and graph $U G$. Any blossom $B$ in a Hungarian subgraph contains an even number of slot vertices $s_{1}, s_{2}$ (part $(i i)$ ) and an odd number of vertices in total. So $B$ contains an odd number of $J$-nodes. Suppose $B$ is augmenting. Part $(i)$ now implies $B$ has $\geq 3 J$-nodes. $\mathcal{P}$ schedules the jobs of $B$ in the time slots of $B$. Clearly they use $\geq 3 / 2$ units of active time.

Next consider arbitrary length jobs and graph $M G$. Let $B$ be an augmenting blossom in $U G$. Lemma 2 shows $B$ has an alternating cycle $C$ of $\geq 5$ edges.

Since $|C|$ is odd, $C$ contains an odd number of edges of the form $s_{1} s_{2}$. Each such $s_{i}$ is adjacent in $C$ to a $J_{U}$-vertex. The corresponding edge has weight $1 / 2$ in $T(M)$, so slot $s$ has (exactly) $1 / 2$ unit of active time in $\mathcal{P}$. If $C$ has $\geq 3$ such edges $s_{1} s_{2}$ then the slots of $B$ have $\geq 3 / 2$ units of active time in $\mathcal{P}$. So suppose $C$ contains exactly one $s_{1} s_{2}$ edge.

The path in $C$ that avoids $s_{1} s_{2}$ must contain some slot vertex $t_{1}, t \in S$ (recall the definition of $M G)$. Since $t_{1} t_{2} \notin C, t_{1}$ is adjacent to two $J_{U}$-vertices in $C$. The corresponding edges have weight $1 / 2$ in $T(M)$. So slot $t$ has 1 unit of active time and $s$ has $1 / 2$, giving the desired $3 / 2$ units. 
Theorem 12 For $B=2$ and a set of jobs $J$, the minimum active time in a schedule permitting preemption only at integer times is $\leq 4 / 3$ times that of one allowing arbitrary preemptions.

Remark: The theorem holds for unit jobs, and for jobs of arbitrary length $\ell_{j}$ when time is slotted, i.e., a schedule with preemptions only at integer times is allowed to execute a length $\ell_{j}$ job in $\ell_{j}$ distinct time slots, but no more. If time is continuous the ratio of the theorem can approach the trivial bound of $B=2$ when job lengths are arbitrary. For example consider a length $\ell$ job with release time 0 and deadline $\ell^{2}$, and unit jobs $j=0, \ldots, \ell-1$ with release time $\ell j$ and deadline $\ell j+1$. The ratio $(2 \ell-1) / \ell$ approaches 2 .

Proof: An augmenting blossom increases the size of the 2-matching by $1 / 2$. Recalling the objective function of LP (7) we see this increases the number of inactive time units by $1 / 2$, i.e., it decreases the number of active time units by $1 / 2$.

Our optimal preemptive schedule $\mathcal{P}$ is constructed from $T(M)$, where the matching $M$ corresponds to an optimal schedule $\mathcal{N}$ with preemptions limited to integer times (recall Lemma 1). Let $M$ have $\pi$ augmenting blossoms and let $\mathcal{P}$ have $\alpha$ active time units. So $\mathcal{N}$ has $\alpha+\pi / 2$ active time units.

The preceding lemma (part (iii)) shows $\alpha \geq(3 / 2) \pi$. Thus the number of active time units in $\mathcal{N}$ exceeds the number in $\mathcal{P}$ by a factor

$$
(\alpha+\pi / 2) / \alpha=1+(\pi / 2) / \alpha \leq 4 / 3 .
$$

\section{Conclusion}

In this paper, we defined a new problem which involves scheduling jobs in batches of size at most $B$. Each job has periods of time within which it must be scheduled and the goal is to minimize the number of active time slots in the schedule. No cost is incurred for slots in which no jobs are scheduled. There is a strong connection between this problem and other classic covering problems such as vertex cover with hard capacities and the $K$-center problem. Another general model of energy consumption might allow for the energy consumption of each active slot to depend on the number of jobs actually assigned to that time slot. At least for the $B=2$ case this can be handled easily, by adapting the matching based solution described in Section 5 .

One could generalize this model further to include other objective functions that measure completion times, tardyness, etc. Furthermore, it would be interesting to consider the online setting in which the entire set of jobs is not known in advance, but jobs arrive over time and are known only when they are released, or perhaps shortly before they are released.

\section{References}

[1] S. Albers. Algorithms for energy saving. In Efficient Algorithms, Springer-Verlag, pp. 173-186, 2009.

[2] S. Albers. Energy-efficient algorithms. in Communications of the ACM, 53(5):86-96, 2010.

[3] S. Albers and A. Antoniadis. Race to idle: new algorithms for speed scaling with a sleep state. in Proceedings of 23rd Annual ACM-SIAM Symposium on Discrete Algorithhms, to appear, 2012 . 
[4] H. Amur, J. Cipra, V. Gupta, M. Kozuch, G. Ganger and K. Schwan. Robust and flexible power-proportional storage. Proceedings of 1st Annual Symp. on Cloud Computing, pp. 217$218,2010$.

[5] M. Babenko, A. Gusakov, and I. Razenshteyn. Triangle-free 2-matchings revisited. Computing and Combinatorics, pp. 120-129, 2010.

[6] P. Baptiste. Batching identical jobs. Mathematical Methods of Operations Research, 52(3):355$367,2000$.

[7] P. Baptiste. Scheduling unit tasks to minimize the number of idle periods: a polynomial time algorithm for offline dynamic power management. Proceedings of 1 th th Annual ACM-SIAM Symposium on Discrete Algorithms, pp. 364-367, 2006.

[8] P. Baptiste, M. Chrobak and C. Dürr. Polynomial time algorithms for minimum energy scheduling. Proceedings of 15th Annual European Symposium on Algorithms, pp. 136-150, 2007.

[9] H. L. Bodlaender and M. R. Fellows. W[2]-hardness of precedence constrained $K$-processor scheduling. Operations Research Letters, 18(2):93-97, 1995.

[10] J. Bar-Ilan, G. Kortsarz and D. Peleg. How to allocate network centers. J. of Algorithms, 15(3):385-415, 1993.

[11] J. Chang, T. Erlebach, R. Gailis and S. Khuller. Broadcast scheduling: algorithms and complexity. Proceedings of 19th Annual ACM-SIAM Symposium on Discrete Algorithms, pp. 473482, 2008.

[12] M. Charikar and S. Khuller. A robust maximum completion time measure for scheduling Proceedings of 17th Annual ACM-SIAM Symposium on Discrete Algorithms, pp. 324-333, 2006.

[13] F. Chudak and D. Williamson. Improved approximation algorithms for capacitated facility location problems. Mathematical Programming, 102(2):207-222, 2005.

[14] J. Chuzhoy and S. Naor. Covering problems with hard capacities. SIAM J. on Computing, 36(2):498-515, 2006.

[15] A. Condotta, S. Knust and N. Shakhlevich. Parallel batch scheduling of equal-length jobs with release and due dates. J. of Scheduling, 13(5):463-677, 2010.

[16] G. Cornuéjols and W. Pulleyblank. A matching problem with side conditions. Discrete Math., 29(2):135-159, 1980.

[17] G. Cornuéjols and W. Pulleyblank. Perfect triangle-free 2-matchings. Math. Programming Study, 13:1-7, 1980.

[18] E. Demaine, M. Ghodsi, M. T. Hajiaghayi, A. S. Sayedi-Roshkar and M. Zadimoghaddam. Scheduling to minimize gaps and power consumption. Proceedings of 19th ACM Symposium on Parallelism in Algorithms and Architectures, pp. 46-54, 2007.

[19] E. Demaine and M. Zadimoghaddam. Scheduling to minimize power consumption using submodular functions. Proceedings of 22nd ACM Symposium on Parallelism in Algorithms and Architectures, pp. 21-29, 2010. 
[20] S. Even and R.E. Tarjan. Network flow and testing graph connectivity. SIAM J. on Computing, 4(4):507-581, 1975.

[21] G. Even, R. Levi, D. Rawitz, B. Schieber, S. Shahar, M. Sviridenko. Algorithms for capacitated rectangle stabbing and lot sizing with joint set up costs. ACM Trans. on Algorithms, 4(3):34-51, 2008 .

[22] T. Erlebach and A. Hall. NP-Hardness of broadcast scheduling and inapproximability of single-source unsplittable min-cost flow. Proceedings of 13th Annual ACM-SIAM Symposium on Discrete Algorithms, pp. 194-202, 2002.

[23] M. Flammini, G. Monaco, L. Moscardelli, H. Shachnai, M. Shalom, T. Tamir and S. Zaks. Minimizing total busy time in parallel scheduling with application to optical networks. International Parallel and Distributed Processing Symposium, pp. 1-12, 2009.

[24] G. N. Frederickson, Scheduling unit-time tasks with integer release times and deadlines. Inf. Proc. Letters, 16(4):171-173, 1983.

[25] M. Fujii, T. Kasami and K. Ninamiya. Optimal sequencing of two equivalent processors. SIAM J. on Computing, 17(4):784-789, 1969.

[26] H. N. Gabow. An efficient reduction technique for degree-constrained subgraph and bidirected network flow problems. Proc. 15th Annual ACM Symp. on Theory of Comput., pp. 448-456, 1983.

[27] H. N. Gabow. An efficient implementation of Edmonds' algorithm for maximum matching on graphs. J. ACM, 23(2):221-234, 1976.

[28] H. N. Gabow. An almost-linear time algorithm for two-processor scheduling. J. ACM, 29(3):766-780, 1982.

[29] R. Gandhi, E. Halperin, S. Khuller, G. Kortsarz and A. Srinivasan. An improved approximation algorithm for vertex cover with hard capacities. J. of Computer and System Sciences, 72(1):1633, 2006.

[30] S. Guha, R. Hassin, S. Khuller and E. Or. Capacitated vertex covering with applications. J. of Algorithms, 48(1):257-270, 2003.

[31] H. N. Gabow. Data structures for weighted matching and nearest common ancestors with linking. Proceedings of 1st Annual ACM-SIAM Symposium on Discrete Algorithms, pp. 434443, 1990.

[32] H. N. Gabow and R. E. Tarjan. Faster scaling algorithms for general graph matching problems. J. ACM, 38(4): 815-853, 1991.

[33] H. N. Gabow and R.E. Tarjan. A linear-time algorithm for a special case of disjoint set union. J. Computer and System Sciences, 30(2):209-221, 1985.

[34] M. Garey and D. S. Johnson. Computers and Intractability: A Guide to the Theory of NPCompleteness. W. H. Freemand and Company, San Francisco, CA, 1979.

[35] M. Garey and D. Johnson. Scheduling tasks with nonuniform deadlines on two processors. J. ACM, 23(3):461-467, 1976. 
[36] M. Garey and D. Johnson. Two processor scheduling with start-times and deadlines. SIAM J. on Computing, 6(3):416-426, 1977.

[37] M. Garey, D. Johnson, B. Simons and R. Tarjan. Scheduling unit-time jobs with arbitrary release times and deadlines. SIAM J. on Computing, 10(2):256-269, 1981.

[38] M. Garey, D. Johnson, R. Tarjan and M. Yannakakis. Scheduling opposing forests. SIAM J. Alg. Disc. Meth., 4(1):72-93, 1983.

[39] Y. Ikura and M. Gimple. Efficient scheduling algorithms for a single batch processing machine. Operations Research Letters, 5(2):61-65, 1986.

[40] S. Irani, R. Gupta and S. Shukla. Algorithms for power savings. ACM Transactions on Algorithms, 3(4): Article 41, 2007.

[41] S. Irani and K. Pruhs. Algorithmic problems in power management. SIGACT News, 36(2): 63-76, 2005.

[42] F. Koehler and S. Khuller. Quick and efficient: fast algorithms for completion time and batch minimization on multiple machines. Manuscript.

[43] S. Khuller and Y. J. Sussman. The capacitated k-center problem. SIAM J. on Discrete Mathematics, 13(30):403-418, 2000.

[44] R. Khandekar, B. Schieber, H. Shachnai and T. Tamir. Minimizing busy time in multiple machine real-time scheduling. Foundations of Software Technology and Theoretical Computer Science Conference, pp. 169-180, 2010.

[45] E. Lawler. Combinatorial Optimization. Holt, Rinehart and Winston, 1976.

[46] R. Levi, D. Shmoys and C. Swamy. LP-based approximation algorithms for capacitated facility location. Proceedings of 10th International Integer Programming and Combinatorial Optimization Conference, pp.206-218, 2004.

[47] M. Li and F. Yao. An efficient algorithm for computing optimal discrete voltage schedules. SIAM J. on Computing, 35(3): 658-671, 2006.

[48] A. López-Ortiz and C.-G. Quimper. A fast algorithm for multi-machine scheduling problems with jobs of equal processing times. Proc. of Symposium on Theoretical Aspects of Computer Science, 2011.

[49] L. Lovász and M.D. Plummer. Matching Theory, North-Holland, 1986.

[50] S. Micali and V.V. Vazirani. An $O(\sqrt{|V|}|E|)$ algorithm for finding maximum matching in general graphs. Proceedings of 21st Annual IEEE Symposium on Foundations of Computer Science, pp. 17-27, 1980.

[51] M. Mucha and P. Sankowski. "Maximum Matchings via Gaussian Elimination". Proceedings of 45th Annual IEEE Symposium on Foundations of Computer Science, pp. 248-255, 2004.

[52] B. Saha. Approximation algorithms for resource allocation. Ph.D. thesis, University of Maryland, August (2011). 
[53] P. Sankowki. Faster dynamic matchings and vertex connectivity. Proc. 18th Annual SODA, 118-126, 2007.

[54] B. Simons. Multiprocessor scheduling of unit-time jobs with arbitrary release times and deadlines. SIAM J. on Computing, 12(2):294-299, 1983.

[55] B. Simons and M. Warmuth. A fast algorithm for multiprocessor scheduling of unit length jobs. SIAM J. on Computing, 18(4):690-710, 1989.

[56] P. Winkler and L. Zhang. Wavelength assignment and generalized interval graph coloring. Proceedings of the 14th ACM-SIAM Symposium on Discrete Algorithms, pp. 830-831, 2003.

[57] L. A. Wolsey. An analysis of the greedy algorithm for the submodular set covering problem. Combinatorica, 2(4):385-393, 1982,

[58] H. Wu and J. Jaffar. Two processor scheduling with real release times and deadlines. ACM Symp. on Parallel Algorithms and Architectures, pp. 127-132, 2002.

[59] F. Yao, A. Demers, S. Shenker. A scheduling model for reduced CPU energy. Proceedings of the 36th Annual IEEE Symposium on Foundations of Computer Science, pp.374-382, 1995. 